\title{
Finite disturbance effect on the stability of a laminar incompressible wake behind a flat plate
}

\author{
By D. RU-SUE KO, T. KUBOTA AND L. LEES \\ California Institute of Technology, Pasadena, California
}

(Received 8 April 1969)

\begin{abstract}
An integral method is used to investigate the interaction between a twodimensional, single frequency finite amplitude disturbance in a laminar, incompressible wake behind a flat plate at zero incidence. The mean flow is assumed to be a non-parallel flow characterized by a few shape parameters. Distribution of the fluctuation across the wake is obtained as functions of those mean flow parameters by solving the inviscid Rayleigh equation using the local mean flow. The variations of the fluctuation amplitude and of the shape parameters for the mean flow are then obtained by solving a set of ordinary differential equations derived from the momentum and energy integral equations. The interaction between the mean flow and the fluctuation through Reynolds stresses plays an important role in the present formulation, and the theoretical results show good agreement with the measurements of Sato \& Kuriki (1961).
\end{abstract}

\section{Introduction}

The use of the infinitesimal disturbance theory of hydrodynamic stability for flow in a two-dimensional wake has been studied by McKoen (1955), Sato \& Kuriki (1961), Gold (1963) and others. The assumption of small disturbance allows the decoupling of the development of the disturbances from the mean flow field, which is independently determined from the steady laminar equations. An exact solution for a steady laminar two-dimensional wake was obtained by Goldstein (1933) with the additional assumptions of a high Reynolds number flow. The linear stability theory further assumes a quasi-parallel mean flow, so that the disturbance equations permit a wave-like solution. A detailed experiment for the wake behind a flat plate in an incompressible flow was reported by Sato \& Kuriki (1961), aiming at clarifying the transition mechanism in a wake. Their measurements confirmed that the initial stage of the laminar-turbulent transition can be described by the linear stability theory. They found that, in this region, the predominant disturbance was two-dimensional and antisymmetric with a frequency corresponding to the one most unstable according to the linear stability theory. However, the agreements are limited to a relatively narrow flow region near the beginning of transition. This limited range of applicability is expected, because the exponentially growing disturbances, no matter how small their initial magnitude may be, will soon invalidate the assumptions pertinent to the 
linearization. Sato \& Kuriki also found that a fairly extensive non-linear region following the linear region existed in the wake.

The following phenomena were observed in this region, and are believed to be consequences of the finite amplitude of the disturbances. (i) Two-dimensional sinusoidal fluctuations, of the same frequency as observed in the linear region, were still prominent. However, their growth deviated from a simple exponential rate; and the amplitude of the disturbance actually decreased in the later stage of this region. (ii) A harmonic at twice the fundamental frequency appeared with measurable amplitude, which was symmetric with respect to the wake axis. (iii) The mean flow velocity and the wake width deviated substantially from the undisturbed laminar wake solution.

An early attempt, to include the effects of a finite amplitude two-dimensional disturbance on the stability of flows between two parallel planes, was reported by Meksyn \& Stuart (1951). The effect of the finite amplitude disturbance was introduced by including the Reynolds-stress term, $\rho \overline{u^{\prime} v^{\prime}}$, in the mean equation of motion. Only a single frequency disturbance was considered, and the generation of the higher harmonics through the non-linear interactions between modes was ignored. Their method of solution was essentially an integral approach. The Reynolds stress was evaluated in terms of a mean flow parameter $U_{0}^{\prime \prime} / U_{0}^{\prime}$, under the assumption that the distribution of the disturbance was given by the solution of the linearized Orr-Sommerfeld equation, where $U_{0}(y)$ is the mean flow velocity and the prime denotes differentiation with respect to $y$. Using such an approach, Meksyn \& Stuart estimated the effect of the finite amplitude disturbance on the critical Reynolds number for plane Poiseuille flow. As was to be expected, the results showed that the critical Reynolds number decreased as the amplitude of the disturbances increased. Stuart (1956) gave a more rigorous formulation. The method was applied to the non-linear instability of plane Couette flow by Kuwabara (1967), who used the Galerkin's method to determine the mean flow and the disturbance.

An enlarged and more general formulation along this line of approach was given by Stuart (1958). In this paper, he discussed extensively the role of the Reynolds stress in determining the stability of parallel flows and the physical processes associated with the non-linearity as the disturbances grow from an infinitesimal to a finite amplitude. A Fourier series expansion was assumed for the disturbance, and an assumption of constant wave velocity $c_{r}$ for all the Fourier components was implied. It in turn gave the expression for the Reynolds stress, which appeared in the mean equation of motion linking the mean flow and the disturbance. An approximate energy method was used, in which the dominant non-linear interaction was assumed to be that between the mean flow and the fundamental component of the disturbance. The distribution of the disturbance was again taken from the solution of the linearized equation. The governing equation for the evolution of the amplitude as a function of time was then obtained from the integrated disturbance energy equation. This amplitude equation turned out to be of the same form as given without derivation by Landau (1944). One most important result of Stuart's analysis is the existence of an equilibrium state when the non-linear effect is introduced. The same method was 
applied to the flow in a small gap between rotating cylinders. Good agreement of the torque required to maintain the cylinders in motion with Taylor's (1923) measurements was indicated.

The basic ideas of the present approach have been extracted from these works. The important difference, however, is that the streamwise variation of the mean flow is considered simultaneously with the evolution of the disturbances.

\section{Formulation of the problem}

\subsection{Governing differential equations}

For two-dimensional flat-plate wake, let $x$ be non-dimensional distance along the wake axis measured from the trailing edge, and $y$ the non-dimensional distance from the wake axis. Correspondingly, $u$ and $v$ represent the nondimensional velocity components. Here the reference quantities for the nondimensionalization are chosen to be the free stream conditions and the physical plate length $L$. Then the Navier-Stokes equations in two dimensions can be written as:

$$
\left.\begin{array}{l}
u_{x}+v_{y}=0 \\
u_{t}+u u_{x}+v u_{y}=-p_{x}+(1 / R)\left(u_{x x}+u_{y y}\right) \\
v_{t}+u v_{x}+v v_{y}=-p_{y}+(1 / R)\left(v_{x x}+v_{y y}\right)
\end{array}\right\}
$$

where $R$ is the Reynolds number based on the reference quantities, i.e. $R=U L / \nu$. Now, the flow is divided into a mean part independent of time and a fluctuating part with zero mean, i.e.

$$
\left.\begin{array}{l}
u(x, y, t)=\bar{u}(x, y)+u^{\prime}(x, y, t) \\
v(x, y, t)=\bar{v}(x, y)+v^{\prime}(x, y, t) \\
p(x, y, t)=\bar{p}(x, y)+p^{\prime}(x, y, t)
\end{array}\right\}
$$

where the bar indicates a time average according to

$$
\bar{Q}=\frac{1}{T} \int_{-\frac{1}{2} T}^{\frac{1}{2} T} Q d t .
$$

The fluctuations are assumed to be periodic in time with period $T$, and hence

$$
\overline{u^{\prime}}=\overline{v^{\prime}}=\overline{p^{\prime}}=0 \text {. }
$$

For simplicity, the time dependence of the fluctuating quantities will be assumed to be of the form $\sim e^{i n \omega t}$. Here the frequency $\omega=2 \pi / T$ is taken to be a real number, and $n$ is an integer.

Substituting (2.2) into (2.1), and averaging over time, we obtain for the mean flow:

$$
\left.\begin{array}{l}
\bar{u}_{x s}+\bar{v}_{y}=0 \\
\overline{u u}_{x}+\overline{v u}_{y}+\left(\overline{u^{\prime 2}}\right)_{x}+\left(\overline{u^{\prime} v^{\prime}}\right)_{y}=-\bar{p}_{x}+(1 / R)\left(\bar{u}_{x x}+\bar{u}_{y y}\right), \\
\overline{u v}_{x}+\overline{v v}_{y}+\left(\overline{u^{\prime} v^{\prime}}\right)_{x}+\left(\overline{v^{\prime 2}}\right)_{y}=-\bar{p}_{y}+(1 / R)\left(\bar{v}_{x x}+\bar{v}_{y y}\right) .
\end{array}\right\}
$$


By subtracting (2.3) from (2.1), we obtain:

$$
\left.\begin{array}{l}
u_{x}^{\prime}+v_{y}^{\prime}=0, \\
u_{t}^{\prime}+\bar{u} u_{x}^{\prime}+u^{\prime} \bar{u}_{x}+\bar{v} u_{y}^{\prime}+v^{\prime} \bar{u}_{y}+\chi_{1}=-p_{x}^{\prime}+(1 / R)\left(u_{x x}^{\prime}+u_{y y}^{\prime}\right), \\
v_{t}^{\prime}+\bar{u} v_{x}^{\prime}+u^{\prime} \bar{v}_{x}+\bar{v} v_{y}^{\prime}+v^{\prime} \bar{v}_{y}+\chi_{2}=-p_{y}^{\prime}+(1 / R)\left(v_{x x}^{\prime}+v_{y y}^{\prime}\right),
\end{array}\right\}
$$

where

$$
\begin{aligned}
& \chi_{1}=u^{\prime} u_{x}^{\prime}+v^{\prime} u_{y}^{\prime}-\left(\overline{u^{\prime} v^{\prime}}\right)_{y}-\left(\overline{u^{\prime 2}}\right)_{x}, \\
& \chi_{2}=u^{\prime} v_{x}^{\prime}+v^{\prime} v_{y}^{\prime}-\left(\overline{u^{\prime} v^{\prime}}\right)_{x}-\left(\overline{v^{\prime 2}}\right)_{y} .
\end{aligned}
$$

For the wake flow behind a flat plate, except in the immediate vicinity of the trailing edge, the boundary layer type approximation is quite satisfactory for a relatively large Reynolds number. In the experiment of Sato \& Kuriki, the Reynolds numbers are of the order of $10^{4}$ and higher. We shall therefore consider flows of large Reynolds number, and apply the boundary layer approximation to the mean flow, which implies:

(i) $\bar{v} / \bar{u}=O\left(R^{-\frac{1}{2}}\right) \ll 1$,

(ii) $b$, the non-dimensional wake half-width much less than 1 ,

(iii) $\frac{\partial}{\partial x} / \frac{\partial}{\partial y}=O\left(R^{-\frac{1}{2}}\right) \ll 1$.

With these approximations, if the magnitude of the fluctuation is further assumed to be infinitesimal, $(2.3)$ reduces to the steady laminar wake equations,

$$
\left.\begin{array}{l}
\bar{u}_{x}+\bar{v}_{y}=0 \\
\overline{u u}_{x}+\overline{v u}_{y}=-\bar{p}_{x}+(1 / R) \bar{u}_{y y} \\
\bar{p}_{y}=0
\end{array}\right\}
$$

where $\bar{p}_{x}$ may be set equal to zero with the boundary condition that $\bar{p}_{x}=0$ as $y \rightarrow \infty$. Equations (2.5) were first solved by Goldstein, who joined a far-wake solution to a series expansion solution for the near-wake. This solution of (2.5) will be referred to as the 'pure laminar wake' solution in this paper.

The role of the finite amplitude disturbances can be seen clearly from (2.3). As the amplitude grows, the Reynolds-stress terms become comparable with the remaining terms in the equation. The rapid change in the mean flow observed experimentally in the non-linear region indicates that the Reynolds-stress terms dominate. If $A$ denotes a measure of the amplitude of the fluctuation, we will expect $A^{2} \sqrt{ } R$ to be of the order one or higher in the non-linear region. In other words, when $A^{2}$ becomes $O(1 / \sqrt{ } R)$, the effect of the disturbance on the mean flow can no longer be ignored. Hence, for the non-linear theory, we will keep the terms resulting from the fluctuating quantities without exact specification of their relative magnitudes as compared with the remaining terms in the mean flow equations.

Eliminating the pressure term from (2.3), we obtain:

$$
\begin{aligned}
\frac{\partial}{\partial y}\left(\overline{u u}_{x}+\overline{v u}_{y}\right) & -\frac{\partial}{\partial x}\left(\overline{u v}_{x}+\overline{v v}_{y}\right)+\frac{\partial}{\partial y}\left(\left({\overline{u^{\prime 2}}}_{x}-\left({\overline{v^{\prime 2}}}_{x}\right)\right.\right. \\
+ & \frac{\partial^{2}}{\partial y^{2}}\left(\overline{u^{\prime} v^{\prime}}\right)-\frac{\partial^{2}}{\partial x^{2}} \overline{\left(u^{\prime} v^{\prime}\right)}=\frac{1}{R} \frac{\partial}{\partial y}\left(\bar{u}_{x x}+\bar{u}_{y y}\right)-\frac{1}{R} \frac{\partial}{\partial x}\left(\bar{v}_{x x}+\bar{v}_{y y}\right) .
\end{aligned}
$$


The underlined terms in the above equation may be neglected by making the boundary layer approximation. Then we have

$$
\left.\left.\frac{\partial}{\partial y}\left[\overline{u u}_{x}+\overline{v u}_{y}+\overline{\left(u^{\prime} v^{\prime}\right.}\right)_{y}+\overline{\left(u^{\prime 2}\right.}-\overline{v^{\prime 2}}\right)_{x}\right]=\frac{1}{R} \frac{\partial}{\partial y} \bar{u}_{y y} .
$$

Integrating $y$ from the edge with the assumption of no disturbance at the edge, we have

$$
\overline{u u}_{x}+\overline{v u_{y}}+\left(\overline{\left(u^{\prime} v^{\prime}\right.}\right)_{y}+\left(\overline{u^{\prime 2}}-\overline{v^{\prime 2}}\right)_{x}=\frac{1}{R} \bar{u}_{y y}
$$

In principle, the complete set of the governing partial differential equations may be solved for any given flow conditions. However, it would be a difficult numerical task, that would provide little understanding of the non-linear mechanism in the wake. Therefore, in an attempt to bring out the essential effects in the non-linear region, the approximate integral method is adopted for the present investigation. In the simplest version of the integral method, the flow is required to satisfy the conservation equations of mean momentum, mean energy and fluctuation energy in integral form. These equations then lead to three ordinary differential equations for three parametric functions characterizing the mean flow and the fluctuation.

\subsection{Integral equations}

The integral equations of the mean flow are obtained by integrating over the lateral co-ordinate $y$. The equations are then reduced to ordinary differential equations in $x$. They are as follows:

Mean momentum equation

$$
\frac{d}{d x} \int_{-\infty}^{\infty}\left(\bar{u}^{2}-\bar{u}+\overline{u^{\prime 2}}-\overline{v^{2}}\right) d y=0
$$

Mean mechanical energy equation

$$
\begin{aligned}
& \frac{d}{d x} \int_{-\infty}^{\infty} {\left[\frac{1}{2}\left(\bar{u}^{3}-\bar{u}\right)+\bar{u}\left(\overline{u^{\prime 2}}-\overline{v^{\prime 2}}\right)\right] d y } \\
&\left.=\int_{-\infty}^{\infty} \overline{\left(u^{\prime 2}\right.}-\overline{v^{\prime 2}}\right) \frac{\partial \bar{u}}{\partial x} d y+\int_{-\infty}^{\infty} \overline{u^{\prime} v^{\prime}} \frac{\partial \bar{u}}{\partial y} d y-\frac{1}{R} \int_{-\infty}^{\infty}\left(\frac{\partial \bar{u}}{\partial y}\right)^{2} d y
\end{aligned}
$$

An additional equation for the fluctuation is provided by the energy equation of the fluctuation, which is obtained by multiplying the second equation of (2.4) by $u^{\prime}$, and the third one by $v^{\prime}$, and adding. This equation is then integrated over $y$ and averaged over a period $T$. It yields the following:

Mean fluctuation energy equation

$$
\begin{aligned}
& \frac{1}{2} \frac{d}{d x} \int_{-\infty}^{\infty}\left[\bar{u}\left(\overline{u^{\prime 2}}+\overline{v^{\prime 2}}\right)+\overline{u^{\prime 3}}+\overline{u^{\prime} v^{\prime 2}}+\overline{2 u^{\prime} p^{\prime}}\right] d y \\
& \left.=-\int_{-\infty}^{\infty} \overline{\left(u^{\prime 2}\right.}-\overline{v^{\prime 2}}\right) \frac{\partial \bar{u}}{\partial x} d y-\int_{-\infty}^{\infty} \overline{u^{\prime} v^{\prime}}\left(\frac{\partial \bar{u}}{\partial y}+\frac{\partial \bar{v}}{\partial x}\right) d y \\
& \left.+\frac{1}{2} \frac{1}{R} \frac{d^{2}}{d x^{2}} \int_{-\infty}^{\infty} \overline{\left(u^{\prime 2}\right.}+\overline{\left.v^{\prime 2}\right)} d y-\frac{1}{R} \int_{-\infty}^{\infty} \overline{\left[\left(\frac{\partial u^{\prime}}{\partial x}\right)^{2}\right.}+\overline{\left(\frac{\partial u^{\prime}}{\partial y}\right)^{2}}+\overline{\left(\frac{\partial v^{\prime}}{\partial x}\right)^{2}}+\overline{\left(\frac{\partial v^{\prime}}{\partial y}\right)^{2}}\right] d y
\end{aligned}
$$


The term $\partial \bar{v} / \partial x$ may be neglected as compared with $\partial \bar{u} / \partial y$, when using the boundary layer approximation for the mean flow. Furthermore, the term involving the second derivative in $x$ represents the streamwise conduction of the fluctuation energy, which is neglected in accordance with the boundary layer approximation.

The advantage of the integral equations is the explicit display of the energy exchange mechanism, because of the conservation forms. In (2.9), the left-hand side terms represent the variation of the mechanical energy associated with the mean flow in the flow direction, while the left-hand side terms in (2.10) give the variation of energy associated with the fluctuations. The transfer of energy between the mean flow and the fluctuations is represented by the two Reynoldsstress terms appearing on the right-hand side of both equations, but with opposite sign. The remaining terms on the right-hand side of both equations represent the viscous dissipation effect.

\subsection{Shape assumption for the mean flow}

It is the main implication of using an integral method approach, that the unknowns may be approximated by a few shape parameters, which will in turn be determined by the integral equations. For the sake of simplicity, the mean velocity profiles are assumed to be characterized by two parameters: the mean velocity defect, $w_{c}(x)=1-\bar{u}(x, 0)$, and the wake half-width, $b(x)$. The mean velocity is then given by

$$
\bar{u}(x, y)=1-w_{c}(x) U^{*}\left(y^{*}\right)
$$

where $y^{*}=y / b(x) . U^{*}\left(y^{*}\right)$ will be assumed known from the experiments, or some other means. This complete similarity of the mean flow is not quite valid in view of the experimental results of Sato \& Kuriki, where overshoot of the mean velocity at some stations was indicated. However, the profiles measured are generally close to a Gaussian distribution, and, therefore, (2.11) is a fairly good approximation throughout the transition region. A better approximation to the mean velocity can be obtained by introducing additional shape parameters, such that

$$
\frac{1-\bar{u}(x, y)}{w_{c}(x)}=U^{*}\left(y^{*}, H_{1}(x), H_{2}(x), \ldots\right) \text {. }
$$

These additional unknown parameters require additional governing equations, which may easily be obtained by using the higher moment equations.

In (2.8), (2.9) and (2.10), we change the independent variables from $(x, y)$ to $\left(x, y^{*}\right)$ according to the following rules:

$$
\left.\begin{array}{rl}
x & =x, \quad y^{*}=\frac{y}{b(x)}, \\
\frac{\partial}{\partial x} & =\frac{\partial}{\partial x}-\frac{y^{*}}{b} \frac{d b}{d x} \frac{\partial}{\partial y^{*}}, \quad \frac{\partial}{\partial y}=\frac{1}{b} \frac{\partial}{\partial y^{*}}
\end{array}\right\}
$$

Then, with $u^{*}=u^{\prime} / w_{c}, v^{*}=v^{\prime} / w_{c}$, and $p^{*}=p^{\prime} / w_{c}^{2}$, together with (2.11), (2.8) and (2.9) become

$$
\frac{d}{d x}\left\{b w_{c}\left[\left(\beta_{1}-\beta_{2} w_{c}\right)-2 w_{c}\left(I_{1}-I_{2}\right)\right]\right\}=\mathbf{0},
$$


and

$$
\frac{d}{d x}\left\{b w_{c}^{2}\left[\beta_{2}-\beta_{3} w_{c}-4 w_{c}\left(I_{3}-I_{4}\right)\right]\right\}=4 w_{c}^{3} \frac{d b}{d x} I_{5}-4 b w_{c}^{2} \frac{d w_{c}}{d x}\left(I_{3}-I_{4}\right)-2 w_{c}^{3} I_{6}-\frac{2 w_{c}^{2}}{R b} \beta_{4}
$$

where

$$
\begin{aligned}
& \beta_{1}=\int_{0}^{\infty} U^{*} d y^{*}, \quad \beta_{2}=\int_{0}^{\infty} U^{* 2} d y^{*} \\
& \beta_{3}=\int_{0}^{\infty} U^{* 3} d y^{*}, \quad \beta_{4}=\int_{0}^{\infty}\left(\frac{\partial U^{*}}{\partial y^{*}}\right)^{2} d y^{*},
\end{aligned}
$$

and

$$
\begin{aligned}
& I_{1}=\frac{1}{2} \int_{0}^{\infty} \overline{u^{* 2}} d y^{*}, \\
& I_{2}=\frac{1}{2} \int_{0}^{\infty} \overline{v^{* 2}} d y^{*}, \\
& I_{3}=\frac{1}{2} \int_{0}^{\infty} U^{*} \overline{u^{* 2}} d y^{*}, \\
& I_{4}=\frac{1}{2} \int_{0}^{\infty} U^{*} v^{* 2} d y^{*}, \\
& I_{5}=\frac{1}{2} \int_{0}^{\infty} y^{*} \frac{\partial U^{*}}{\partial y^{*}}\left(\overline{u^{* 2}}-\overline{v^{* 2}}\right) d y^{*}, \\
& I_{6}=\int_{0}^{\infty} \overline{u^{*} v^{*}} \frac{\partial U^{*}}{\partial y^{*}} d y^{*} .
\end{aligned}
$$

There will be an additional term of the form

$$
\left[-b w_{c}^{3} \int_{0}^{\infty} \frac{\partial U^{*}}{\partial x}\left(\overline{u^{* 2}}-\overline{v^{* 2}}\right) d y^{*}\right]
$$

on the right-hand side of (2.15), when the expression (2.12) is used instead of (2.11). Without losing the main features of the present approach, we will use (2.11) throughout this paper. In this case the $\beta_{i}$ 's are constants.

Equation (2.10) can be written as

$$
\begin{aligned}
\frac{d}{d x}\left[b w _ { c } ^ { 2 } \left\{\left(I_{1}+I_{2}\right)-w_{c}\left(I_{3}\right.\right.\right. & \left.\left.\left.+I_{4}\right)+w_{c}\left(I_{7}+I_{9} / 2\right)\right\}\right] \\
& =w_{c}^{2} I_{6}-2 w_{c}^{3} \frac{d b}{d x} I_{5}+2 b w_{c}^{2} \frac{d w_{c}}{d x}\left(I_{3}-I_{4}\right)-\frac{w_{c}^{2}}{R b} I_{8}
\end{aligned}
$$

where

$$
\begin{aligned}
& I_{7}=\int_{0}^{\infty} \overline{u^{*} p^{*}} d y^{*} \\
& I_{8}=\int_{0}^{\infty}\left[\overline{\left(\frac{\partial u^{*}}{\partial x^{*}}\right)^{2}}+\overline{\left(\frac{\partial u^{*}}{\partial y^{*}}\right)^{2}}+\overline{\left(\frac{\partial v^{*}}{\partial x^{*}}\right)^{2}}+\overline{\left(\frac{\partial v^{*}}{\partial y^{*}}\right)^{2}}\right] d y^{*}, \\
& I_{9}=\int_{0}^{\infty} \overline{\left(\overline{u^{* 3}}+\overline{u^{*} v^{* 2}}\right) d y^{*}}
\end{aligned}
$$


The viscous dissipation integral is given approximately by $I_{8}$ after replacing the $x$ derivatives appearing inside the last integral of $(2.10)$ by its local values, i.e.

$$
\frac{\partial}{\partial x} \approx \frac{1}{b} \frac{\partial}{\partial x^{*}} \text { for } \quad x^{*}=\frac{x-x_{0}}{b(x)}
$$

with $x_{0}$ being a reference station near $x$. It may be shown that the neglected terms are of the order of the square of the dimensionless fluctuation amplitude $A$ (defined in \$2.4), and this approximation introduces the same order of error as neglecting the conduction term in (2.10). Equation (2.14a) can be immediately integrated to give:

$$
b w_{c}\left[\left(\beta_{1}-\beta_{2} w_{c}\right)-2 w_{c}\left(I_{1}-I_{2}\right)\right]=\frac{C_{D}}{2}=\frac{0 \cdot 664}{\sqrt{R}},
$$

where the integration constant has been obtained by assuming a laminar flow over the flat plate. It should be noted that the effect of body shape appears only through the drag coefficient $C_{D}$. Equations (2.14), (2.15) and (2.16) provide the governing equations for the interaction between the mean flow and the fluctuations.

\subsection{Shape assumptions for the fluctuation}

The results of Sato \& Kuriki have demonstrated the dominancy of a single frequency fluctuation. Therefore, as a first-order approximation, the fluctuation is assumed to be represented by a single frequency disturbance, and the generation of the higher harmonics is ignored. This assumption implies that the nonlinear quadratic terms $\chi_{1}$ and $\chi_{2}$ in (2.4) are neglected. The remaining equations are then linear in the fluctuating quantities which, when properly normalized by the local mean flow quantities, admit a solution of the form,

$$
\left.\begin{array}{l}
u^{*}=u^{\prime} / w_{c}=\epsilon(x) f_{0}^{\prime}\left(y^{*} ; x\right) \exp \left\{i\left(\alpha^{*} x^{*}-\omega^{*} t^{*}\right)\right\}+\text { conj }, \\
v^{*}=v^{\prime} / w_{c}=-i\left[\epsilon(x) \alpha^{*} f_{0}\left(y^{*} ; x\right) \exp \left\{i\left(\alpha^{*} x^{*}-\omega^{*} t^{*}\right)\right\}-\mathrm{conj}\right], \\
p^{*}=p^{\prime} / w_{c}^{2}=\epsilon(x) p_{0}\left(y^{*} ; x\right) \exp \left\{i\left(\alpha^{*} x^{*}-\omega^{*} t^{*}\right)\right\}+\mathrm{conj},
\end{array}\right\}
$$

where $\alpha^{*}=\alpha b$ is the normalized complex wave-number, $\omega^{*}=\omega b$, a local real angular frequency and $A(x)=\epsilon(x) \exp -\left(\alpha_{i}^{*} x^{*}\right)$ measures the amplitude of the disturbance. The star indicates local variables.

Upon substituting expression (2.17) into (2.4), and neglecting all the nonlocal terms (e.g. $\partial \bar{u} / \partial x$ ), we obtain an equation for the distribution function $f_{0}\left(y^{*}\right)$. To further simplify the numerical analysis, we observe that a wake is dynamically unstable, and the viscous terms may be ignored for large $R$, except for a few occasions in which the viscous terms are needed to smooth out singularity. Therefore, using the expression (2.11) for the mean flow, we obtain the Rayleigh equation for $f_{0}$,

$$
\left(U^{*}-\bar{c}\right)\left(\frac{d^{2}}{d y^{* 2}}-\alpha^{* 2}\right) f_{0}-\frac{d^{2} U^{*}}{d y^{* 2}} f_{0}=0
$$

where

$$
\bar{c}=\frac{1}{w_{c}}\left(1-\frac{\omega^{*}}{\alpha^{*}}\right) \text {. }
$$


The linear stability theory has indicated that an antisymmetric fluctuation is more unstable than a symmetric one as confirmed by the appearance of an antisymmetric disturbance in the experiment of Sato \& Kuriki. Thus, the fluctuation is assumed to be antisymmetric, which satisfies the homogeneous boundary conditions,

$$
\left.\begin{array}{l}
f^{\prime}(0)=0, \\
f^{\prime}\left(y^{*}\right)+\alpha^{*} f\left(y^{*}\right)=0, \text { as } y^{*} \rightarrow \infty .
\end{array}\right\}
$$

Equation (2.18), together with the boundary condition (2.18a), constitutes an eigenvalue problem. For given $w_{c}$, $(2.18)$ can be solved numerically to obtain the eigenvalue and the corresponding eigenfunction $f_{0}\left(y^{*}\right)$. In general, for a given $U^{*}\left(y^{*}\right)$, we have

$$
\left.\begin{array}{l}
\alpha^{*}=\alpha^{*}\left(w_{c}, \omega^{*}\right), \\
f_{0}=f_{0}\left(y^{*} ; w_{c}, \omega^{*}\right)
\end{array}\right\}
$$

which indicate the functional dependence of the eigenvalue and the eigenfunction. Here we consider the spatial mode of linear stability theory, i.e. the disturbance grows or decays spatially, depending on the sign of the imaginary part of $\alpha^{*}$.

The use of a Rayleigh equation with the local mean-velocity profile, to obtain the distributions of the fluctuating components across the wake, may seem to be quite arbitrary without a more rigorous analysis. At the moment, we will just note that this is merely a method of generating the fluctuation profiles, in order to evaluate the required integrals as functions of the mean flow parameters and the amplitude. The integral method does not restrict the means of obtaining the distributions, as long as they are good representations of the true ones. However, the use of the Rayleigh equation at least guarantees the validity in the limit of very small amplitude.

It should be noted that the formulation so far has reduced the integrals $I_{i}$ to a two-parameter representation aside from the direct dependence on the amplitude $A$. Further approximation will now be made to simplify the analysis. We observe that, in general, the fluctuation components are much smaller than the mean-flow component and, since both $I_{1}$ and $I_{2}$ are positive and of the same order, the contributions from the fluctuations in (2.14) may be ignored as a first approximation. This reduces $(2.14)$ to

$$
b=\frac{\frac{1}{2} C_{D}}{w_{c}\left(\beta_{1}-\beta_{2} w_{c}\right)}
$$

Then $\omega^{*}$ becomes a function of $w_{c}$, and the integrals are simplified to be functions of a single parameter $w_{c}$, when the physical angular frequency and the free stream Reynolds number are given. Of course, the validity of this assumption will have to be examined a posteriori. Thus, the integrals can be written in the form,

$$
I_{i}=k_{i}\left(w_{c}\right)|A|^{2}
$$

for $i=1,2, \ldots, 8$. When only the fundamental mode is included, the triple correlations vanish, and hence $I_{9}$ becomes zero. 
Using (2.20), (2.15) and (2.16) can be written as two first-order ordinary differential equations for $w_{c}$ and $|A|^{2}$, which may be solved as an initial value problem. The exact definition of $A$ will now be given by setting

$$
k_{1}+k_{2}=\int_{0}^{\infty}\left[\left|f_{0}^{\prime}\right|^{2}+\left|\alpha^{*}\right|^{2}\left|f_{0}\right|^{2}\right] d y^{*}=1
$$

This definition is appropriate, since it identifies $|A|^{2}$ as the averaged energy of the fluctuating components normalized by the local mean velocity defect.

\subsection{Physical mechanisms of energy balance}

In order to bring out the physical mechanisms of the non-linear interaction between the mean flow and the fluctuation, we need consider only the leading terms in the basic equations. In $\S 3$ these qualitative considerations are supplemented by numerical calculations using the full equations.

The integrated momentum equation (2.14), to the leading order, gives simply

$$
b w_{c} \cong \gamma_{1} R^{-\frac{1}{2}}
$$

where $\gamma_{1}$ is a known constant of order one. The energy integral equations of the mean flow and the fluctuation to the first order, may be written as

$$
\frac{d E_{m}}{d x} \simeq-I_{6} w_{c}^{3}-\frac{\beta_{4} w_{c}^{2}}{R b}
$$

and

$$
\frac{d E_{F}}{d x} \simeq I_{6} w_{c}^{3}-\frac{I_{8} w_{c}^{2}}{R b}
$$

correspondingly. Here $E_{m}$ denotes the integral energy of the mean flow. For small $w_{c}$, since $b w_{c}$ is nearly constant according to (2.23), $E_{m}$ is linearly proportional to $w_{c}$ to the first order. $E_{F}$ is the total integrated fluctuation energy given by

$$
E_{F}=\int_{0}^{\infty}\left(\overline{u^{\prime 2}}+\overline{v^{\prime 2}}\right) d y .
$$

The first terms on the right-hand side of (2.24) and (2.25) are the same, but with an opposite sign. These terms represent an energy transfer between the mean flow and the fluctuations due to the Reynolds stress. For a locally amplified disturbance, the sign of $I_{6}$ is always positive. Therefore, the energy is transferred from the mean flow to the fluctuation through the Reynolds stress. The remaining terms on the right-hand side of both equations represent the effect of viscous dissipation.

Because of the growth of the wake, it is more interesting to examine the velocity defect $w_{c}$ and the total energy density of the fluctuation $E_{T}$ defined by

$$
E_{T}=\frac{E_{F}}{b}=2|A|^{2} w_{c}^{2}
$$

Then, from (2.21), (2.24) and (2.25), we obtain:

$$
\begin{gathered}
\gamma_{2} R^{-\frac{1}{2}} \frac{d w_{c}}{d x}=-k_{6}\left(w_{c}\right) E_{T} w_{c}-\frac{2 \beta_{4} w_{c}^{3}}{\gamma_{1} R^{\frac{1}{2}}} \\
\frac{1}{E_{T}} \frac{d E_{T}}{d x}=\left(\frac{1}{\gamma_{1}}-\frac{E_{T}}{\gamma_{2} w_{c}^{2}}\right) k_{6}\left(w_{c}\right) w_{c}^{2} R^{\frac{1}{2}}-\frac{w_{c}^{2}}{\gamma_{1}}\left(\frac{k_{8}\left(w_{c}\right)}{\gamma_{1}}+\frac{2 \beta_{4}}{\gamma_{2}}\right),
\end{gathered}
$$


where $\gamma_{2}$ is a known constant of order one, which accounts for the proportionality between $w_{c}$ and $E_{m}$.

A qualitative behaviour of the flow field in the non-linear region may be obtained from these two equations. When the amplitude of the fluctuation is small, the first term on the right-hand side of (2.27) is negligible as compared with the laminar viscous dissipation term, and the mean flow is closely approximated by the steady laminar solution, in which $w_{c}$ decreases as $x^{-\frac{1}{2}}$. Since $w_{c}$ changes slowly in this region, the right-hand side of (2.28) is approximately constant. Then, the exponential growth rate of the linear stability theory immediately follows. This region corresponds to the linear region observed by Sato \& Kuriki.

As the amplitude grows, the Reynolds stress term becomes comparable with the viscous term in (2.27), and the mean velocity defect starts deviating from the steady laminar solution. As the fluctuation is further amplified, the Reynolds stress term becomes dominating. The experimentally observed rapid change of the mean velocity and the wake width in the non-linear region may be understood from this consideration. From the solution of the Rayleigh equation we learn that the local amplification rate decreases as $w_{c}$ decreases, if a single fixed frequency fluctuation is followed. Hence, when the fluctuation corresponding to the most unstable frequency in the linear region is taken to represent the fluctuating component, it will approach neutral as $w_{c}$ decreases. Thus, when $w_{c}$ decreases to the value where $k_{6}$ becomes small, the mean flow is expected to have a relatively slow variation, as observed experimentally.

The qualitative behaviour of the fluctuation in the non-linear region may be deduced from (2.28). For $k_{6} \gg R^{-\frac{1}{2}}$, the first term dominates. The appearance of the $E_{T}$ term with a negative sign on the right-hand side of (2.28) is the most interesting feature caused by the growth of the wake. It permits not only a state where $d E_{T} / d x=0$, but also a decreasing $E_{T}$ with $x$ even when the fluctuation under consideration is still amplifying according to the local linear theory $\left(k_{6}>0\right)$. Experimentally, the maximum amplitude of the fundamental mode grows initially, but decreases after reaching a peak value. The solution of our model equation exhibits the same behaviour, since the energy density $E_{T}$ is expected to be indicative of the magnitude of the fluctuation.

The crucial differences of the present problem from the parallel flow analysis of Stuart $(1960)$ and Watson $(1960,1962)$ become evident from the above discussions. The main result of the non-linear analysis of Stuart and Watson is the governing equation for the amplitude of the disturbances, which is of the form

$$
\frac{1}{|A|^{2}} \frac{d|A|^{2}}{d x}=\bar{a}_{0}+\bar{a}_{1}|A|^{2}+\ldots
$$

where $\bar{a}_{n}$ 's are constants. The coefficient $\bar{a}_{0}$ is given by the linear theory with undisturbed laminar mean flow, and $\bar{a}_{1}$ is a result of three effects: the generation of the second harmonic, the correction of the fundamental and the correction to the laminar mean flow. The amplitude $|A|$ in the StuartWatson theory corresponds to the average energy density $E_{T}$ defined by (2.26). Then, an analogous equation of the form of (2.29) results, with $|A|^{2}$ replaced by $E_{r}$. However, the coefficients $\bar{a}_{0}$ and $\vec{a}_{1}$ are no longer constants but functions 
of $x$ through the variation of the mean flow. Since the mean flow is not expanded as the undisturbed laminar flow plus a correction in the present problem, but is lumped together to be determined by the integral equations, the so-called second Landau constant $\bar{a}_{1}$ does have an appreciable magnitude, even when the second harmonic is ignored. The sign of $\bar{a}_{1}$ is opposite to that of $\bar{a}_{0}$. In the case of a parallel flow, a supercritical equilibrium state may exist. However, the continued variation of the mean flow provides the possibility of a decreasing magnitude of the fluctuations before reaching the final equilibrium state, as demonstrated previously.

\section{Results and discussion}

For the purpose of comparison with the experimental results of Sato \& Kuriki, the numerical calculations have been performed corresponding closely to the experimental conditions. In the calculation, the mean velocity $U^{*}\left(y^{*}\right)$ is that used by Sato \& Kuriki in the linear region, i.e.

$$
U^{*}\left(y^{*}\right)=\exp \left(-0.69315 y^{* 2}\right)
$$

Equation (3.1) also gives the exact definition of the half-wake width $b$ as the distance from the wake axis to the half velocity defect point, where $U^{*}=0.5$. In the calculation, too, the frequency of the fundamental mode taken is that in the experiment of Sato \& Kuriki, $730 \mathrm{c} / \mathrm{s}$ at $R=2 \times 10^{5}$. This frequency was found to be the one receiving nearly maximum amplification rate in the linear region given by the temporal mode calculation of Sato \& Kuriki.

With the frequency fixed at this value, the variation of the local amplification rate was determined as a function of the mean velocity defect $w_{c}$ by solving the Rayleigh equation as formulated in (2.4). The result shows that the amplification rate decreases as $w_{c}$ decreases. At $w_{c} \approx 0 \cdot 147, \alpha_{i}^{*}=0$; the given frequency corresponds to a neutrally stable solution according to the linear stability theory. Further decrease of the mean flow parameter $w_{c}$ will make this frequency a damped disturbance $\left(\alpha_{i}^{*}>0\right)$, according to the linear theory.

The integrals $k_{i}$ as functions of $w_{c}$ were then computed from the eigensolutions of the Rayleigh equation. After this preparatory computation, the integral conservation equations $(2.14),(2.15)$ and (2.16) were integrated for each given set of initial conditions to determine the simultaneous evolutions of the mean flow and the fluctuation.

\subsection{Comparison with the experiment}

Most of the experimental results presented by Sato \& Kuriki were obtained with a free-stream velocity $U=10 \mathrm{~m} / \mathrm{sec}$ and a plate length $L=30 \mathrm{~cm}$. These conditions correspond to a free-stream Reynolds number of $2 \times 10^{5}$. From the experimental evidences, the wake was steady and laminar for a short distance from the trailing edge of the plate. The calculations are therefore started at an initial station $x=\zeta_{0}=0.05$ using the appropriate initial conditions. The centre-line velocity defect $w_{c 0}$ at $\zeta_{0}$ can be taken from either the exact solution of Goldstein or the integral solution of an undisturbed laminar wake in the present formula- 
tion. The latter is not very accurate there, since the velocity distribution deviates from the Gaussian as $\zeta_{0}$ decreases. For the present purposes of comparison, it was decided to use $w_{c 0}=0.7$ at $\zeta_{0}=0.05$ from the exact solution of Goldstein, because of the closer agreement with the experiment at that point. Another initial parameter is the initial integrated energy content in the $u^{\prime}$ component, i.e.

$$
E_{u 0}=\left(\frac{1}{b} \int_{0}^{\infty} \overline{u^{\prime 2}} d y\right)_{0}
$$

where the subscript 0 refers to value at $\zeta_{0}$. The value of this parameter depends on the different flow conditions encountered in each experiment, e.g. the free stream turbulence intensity level, the physical dimension of the plate trailing edge, etc. In the present study these two initial parameters were chosen somewhat arbitrarily in performing the following calculations. The effects of each of the initial parameters on the complete solutions will be investigated later.

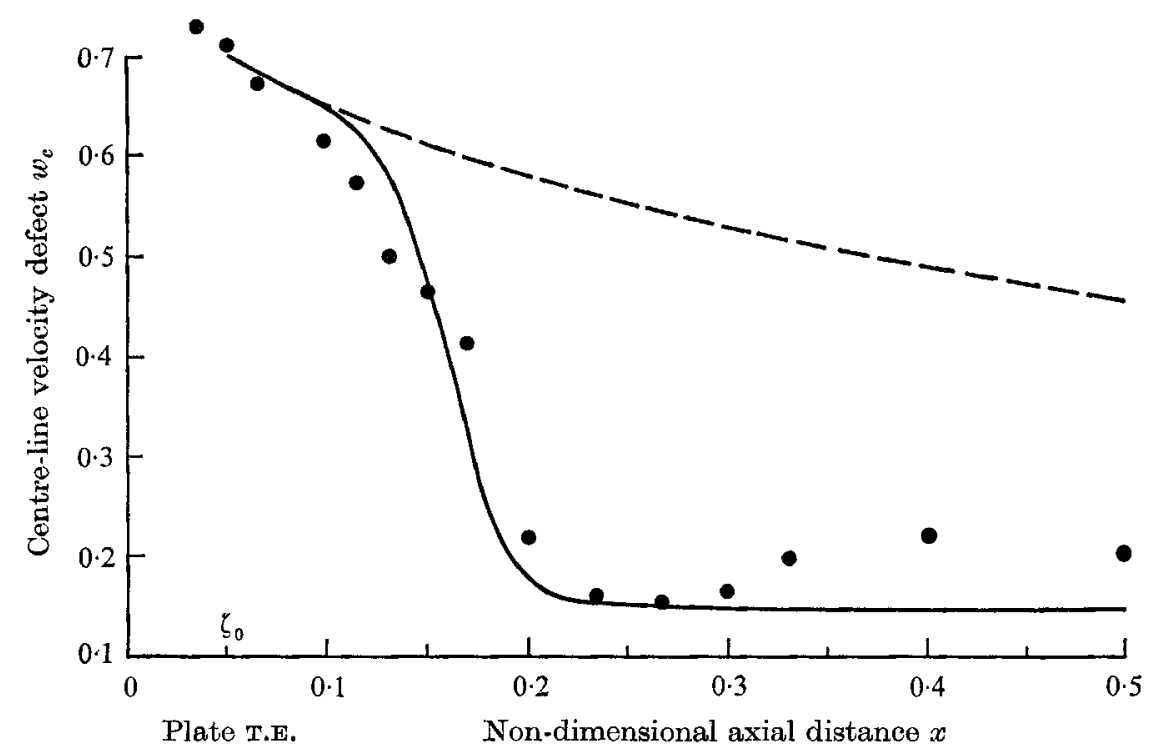

Fraure 1. Comparison of the mean centre-line velocity defect $w_{c}$. measurements of Sato \& Kuriki at $R=2 \times 10^{5}$. at $\zeta_{0}=0.05 . \cdots$, pure laminar wake.

Figure 1 shows a comparison of the measured centre-line velocity defect $w_{c}$ with the present calculation. The value of $E_{u 0}$ was taken as $1 \times 10^{-5}$ in the calculation. The result is quite satisfactory, and seems to provide the explanation for the rapid deviation from the pure laminar wake solution of Goldstein, which is also shown on the same figure for comparison.

Only the results for $x \leqslant 0.5$ are presented, because the three-dimensional effects observed experimentally at larger $x$ are not included in the present formulation. Theoretical calculation for $x>0.5$ stays practically unchanged near the value of $w_{c}=0 \cdot 148$. It is also interesting to note that this asymptotic value of $w_{c}$ corre- 
sponds closely to the value where $\alpha_{i}^{*}=0$. At this value a balance exists between various mechanisms responsible for changing the mean flow. We may note that, although it may be somewhat fortuitous, the measured $w_{c}$ in all the tests has never become smaller than this value before the turbulent region.

Figure 2 shows the comparison of the measured wake half-width with the one calculated by using (2.20). The general trend is still satisfactory, but not as good as $w_{e}$. This disagreement seems to suggest that the approximation used in leading to (2.20) may not be appropriate, if a better calculation is required. In such cases, the exact relation (2.14) has to be used, which includes the contributions from the fluctuation in the momentum integral. An attempt to include this effect will be discussed in \$3.5. Also shown on the same plot is the growth of the undisturbed laminar wake. The strong interaction effect induced by the Reynolds stresses is evident from this comparison, where the wake width has increased by more than a factor of two.

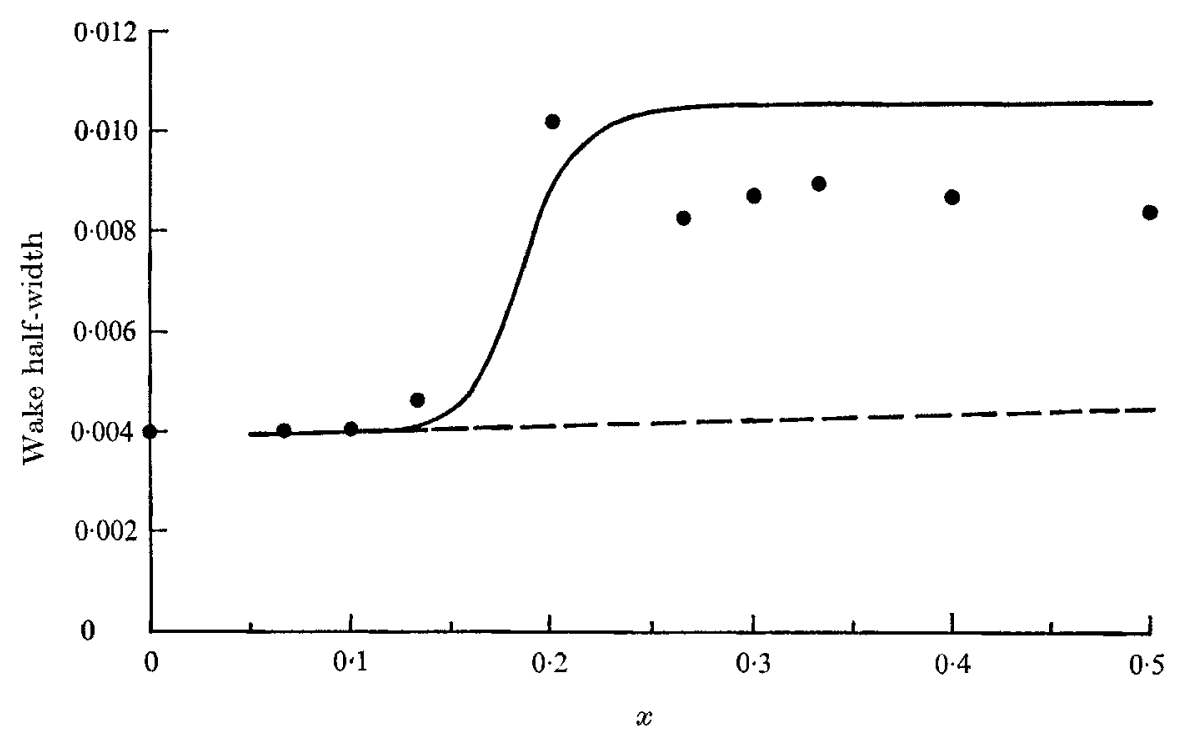

Fiqure 2. Comparison of the wake half-width $b$ (defined as the distance from the axis to the half velocity defect point non-dimensionalized by the plate length) $R=2 \times 10^{5}$, $w_{c 0}=0.7, E_{u 0}=1 \times 10^{-5}$. , measurements of Sato \& Kuriki.

Figure 3 gives the theoretically calculated variation of the integrated fluctuation energy,

$$
E_{u}=\frac{1}{b} \int_{0}^{\infty} \overline{u^{\prime 2}} d y
$$

Since the absolute value of the magnitude of the fluctuating components was not reported by Sato \& Kuriki, a direct comparison with the experiment is not possible. However, the variation of the maximum of $\left(\bar{u}_{f}^{2}\right)^{\frac{1}{2}}$, given by Sato \& Kuriki on an arbitrary scale, shows the similar relative development of the fluctuation. Experimentally, the magnitude of the fundamental mode grows initially according to the exponential law of the linear stability theory, but it 


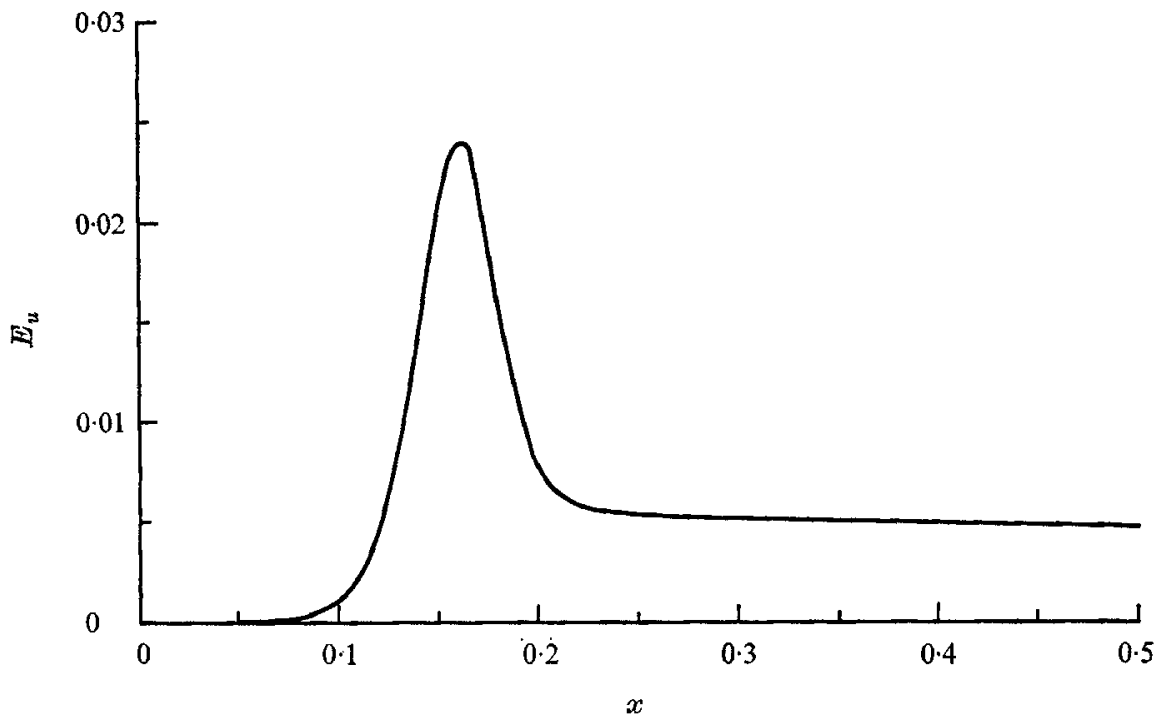

Figure 3. Calculated variation of the integrated fluctuation energy density in the $u^{\prime}$ component $E_{u}$, where $E_{u}=\frac{1}{b} \int_{0}^{\infty} \overline{u^{\prime 2}} d y . R=2 \times 10^{5}, w_{c 0}=0 \cdot 7, E_{u 0}=1 \times 10^{-5}$.

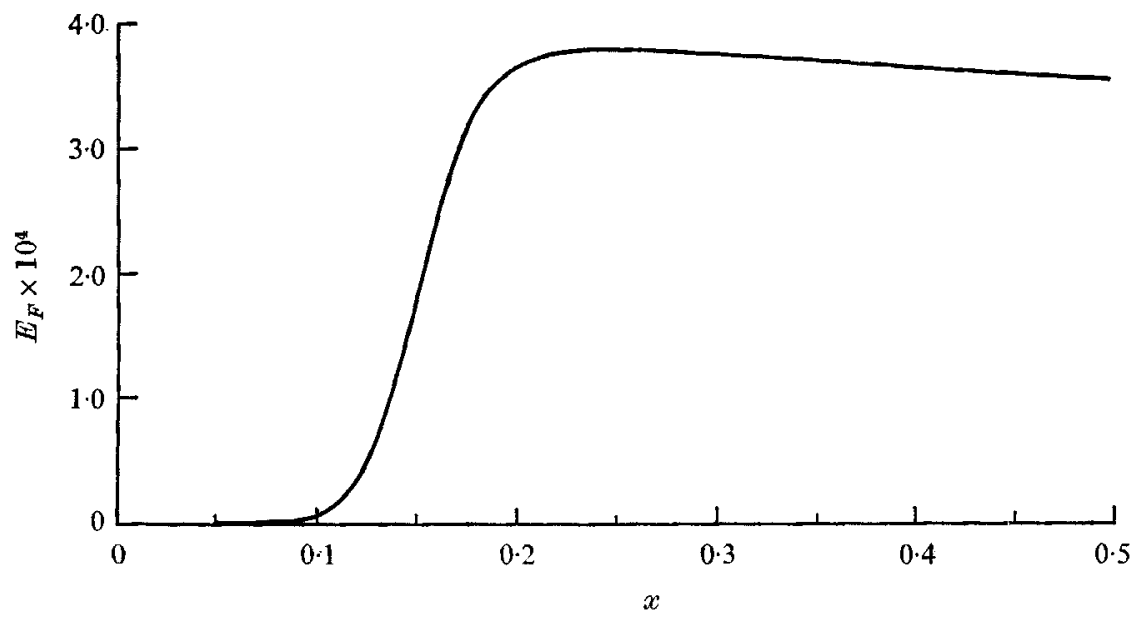

Figure 4. Calculated variation of the total integrated fluctuation energy $E_{F}$, where $\left.E_{F}=\int_{0}^{\infty} \overline{\left(u^{\prime 2}\right.}+\overline{v^{\prime 2}}\right) d y . R=2 \times 10^{5}, w_{c 0}=0.7, E_{u 0}=1 \times 10^{-5}$.

soon reaches a maximum and then decreases. This behaviour, which cannot be explained by the linear stability theory alone, is in agreement with the present calculation. However, it should be pointed out that the rapid decrease of $E_{u}$ does not imply the similar decrease of the total fluctuation energy. This is seen in figure 4 where the total integrated fluctuation energy $E_{F}$ is plotted. The variation of $E_{F}$, is related to the local amplification rate $\alpha_{i}^{*}$, given by the linear theory, with 
some correction due to the variation of the mean flow. The rate of change of the total energy starts out in the linear region with a nearly maximum exponential growth, and decreases as it moves downstream. The total energy reaches an equilibrium value somewhere near $\alpha_{i}^{*}=0$, when the mean flow ceases to vary, and then slowly decays because of the viscous dissipating effect. Because of the growth of the wake, a more appropriate measure of the magnitude of the disturbance is the total energy density $E_{T}$ defined by $E_{T}=E_{F} / b$. The variation of $E_{T}$ is shown in figure 5 . The reason for the relatively much slower variation of the total fluctuating energy density $E_{T}$, as compared with the sharp decrease in $E_{u}$ (figure 3), is shown in figure 6 , where the ratio of the integrated energy content in $u^{\prime}$ to that in $v^{\prime}$,

$$
k_{1} / k_{2}=\int_{0}^{\infty} \overline{u^{\prime 2}} d y^{*} / \int_{0}^{\infty} \overline{v^{\prime 2}} d y^{*}
$$

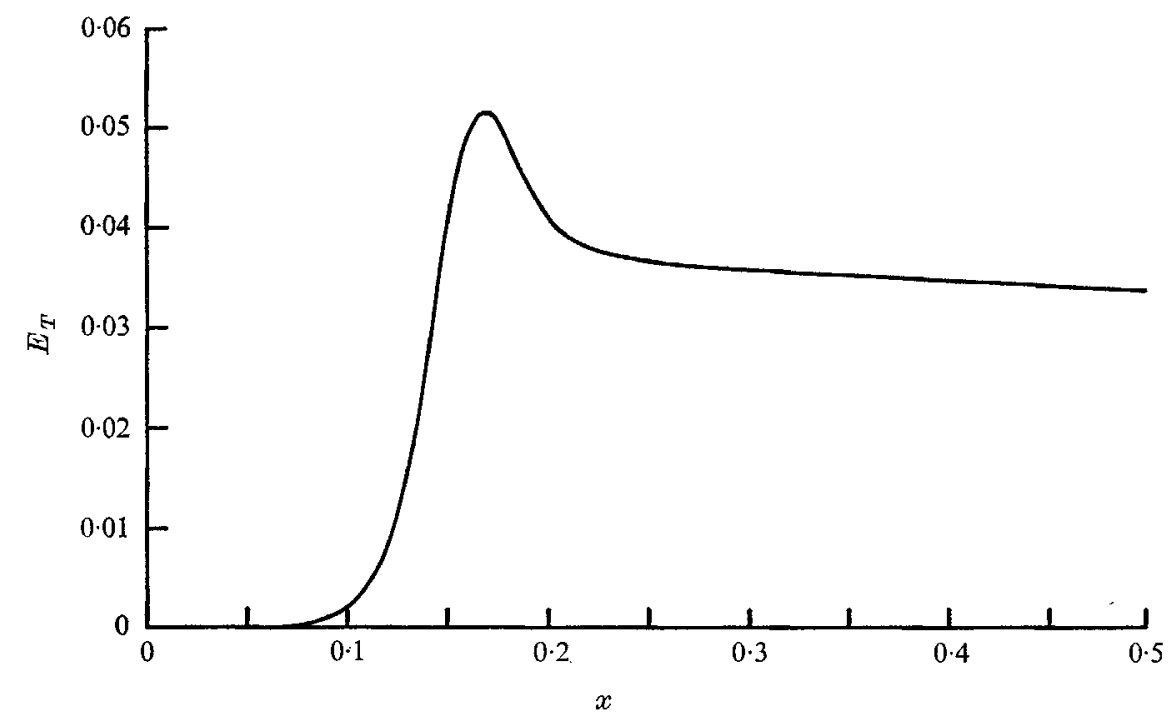

FIGURE 5. Calculated variation of the total integrated fluctuation energy density $E_{T}$, where $\left.E_{T}=\frac{1}{b} \int_{0}^{\infty} \overline{\left(u^{\prime 2}\right.}+\overline{v^{\prime 2}}\right) d y . R=2 \times 10^{5}, w_{c 0}=0 \cdot 7, E_{u 0}=1 \times 10^{-5}$.

is plotted against $w_{c}$. The ratio varies by more than a factor of six for the range of $w_{c}$ encountered here. Therefore, we conclude that the redistribution of the fluctuating energy between the two components $u^{\prime}$ and $v^{\prime}$, together with the change of mean flow, is responsible for the experimentally observed abnormal phenomena.

The non-dimensional wave propagation velocity, taken as the real part of $c^{*}=\omega^{*} / \alpha^{*}$, measured by Sato \& Kuriki, can also be obtained from the present calculation and the comparison is shown in figure 7. In view of the scatter of the experimental data, the agreement shown is acceptable. The variation of the wave speed is again a consequence of the changing mean flow. 


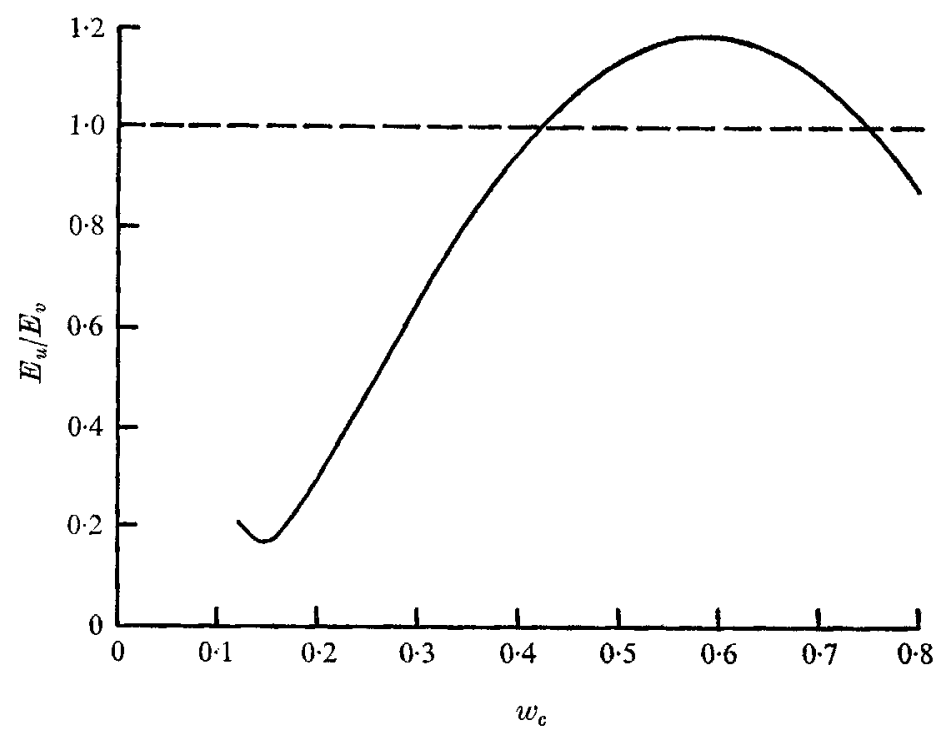

FIGURE 6. Relative energy content in the $u^{\prime}$ and $v^{\prime}$ components as functions of $w_{c}$. Frequency of the fluctuation $=730 \mathrm{cps}$ at $R=2 \times 10^{5}$.

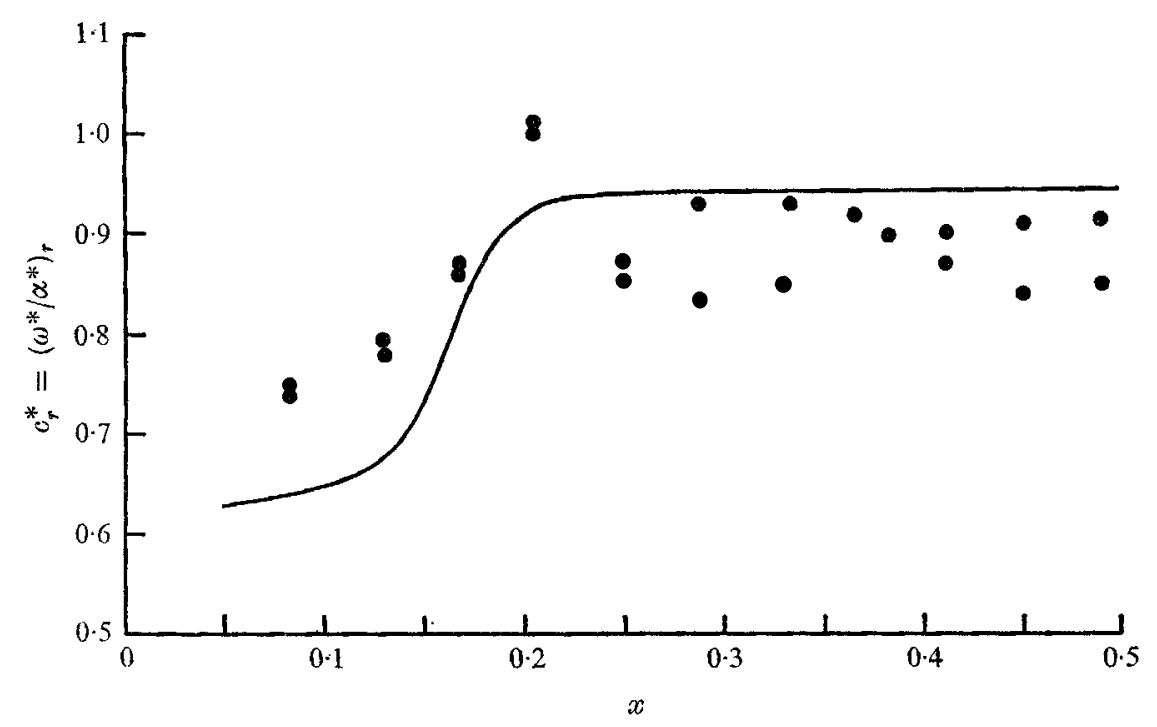

FIGURE 7. Comparison of the non-dimensional wave propagation velocity $c_{r}^{*}=\left(\omega^{*} / \alpha^{*}\right)_{r}$ at $R=2 \times 10^{5}$. $\bigcirc$, measurements of Sato \& Kuriki. —, present calculation.

\subsection{Effects of the initial values}

Although no special attempt has been made in obtaining the theoretical curves discussed in this section to match the experimental data, the arbitrariness in the set of initial values warrants an investigation of their effects. We will study the effects of the two initial values, $w_{c 0}$ and $E_{u 0}$, separately in the following. 
(i) Effects of $w_{c 0}$. Three cases of variation of $w_{c}$ corresponding to the initial values of $w_{c 0}$ being $0.7,0.675$ and 0.6386 , are shown in figure 8 . The value $w_{c 0}=0.6386$ corresponds to the steady laminar wake solution at $\zeta_{0}=0.05$ obtained by setting the fluctuation amplitude equal to zero and integrating (2.15) backward from a far wake solution. The same value of $E_{u 0}$ was used for all three curves, which give qualitatively the same variation of $w_{c}$, and asymptote to the same value of $w_{c}$. The decrease in $w_{c 0}$ results in the decrease in the maximum

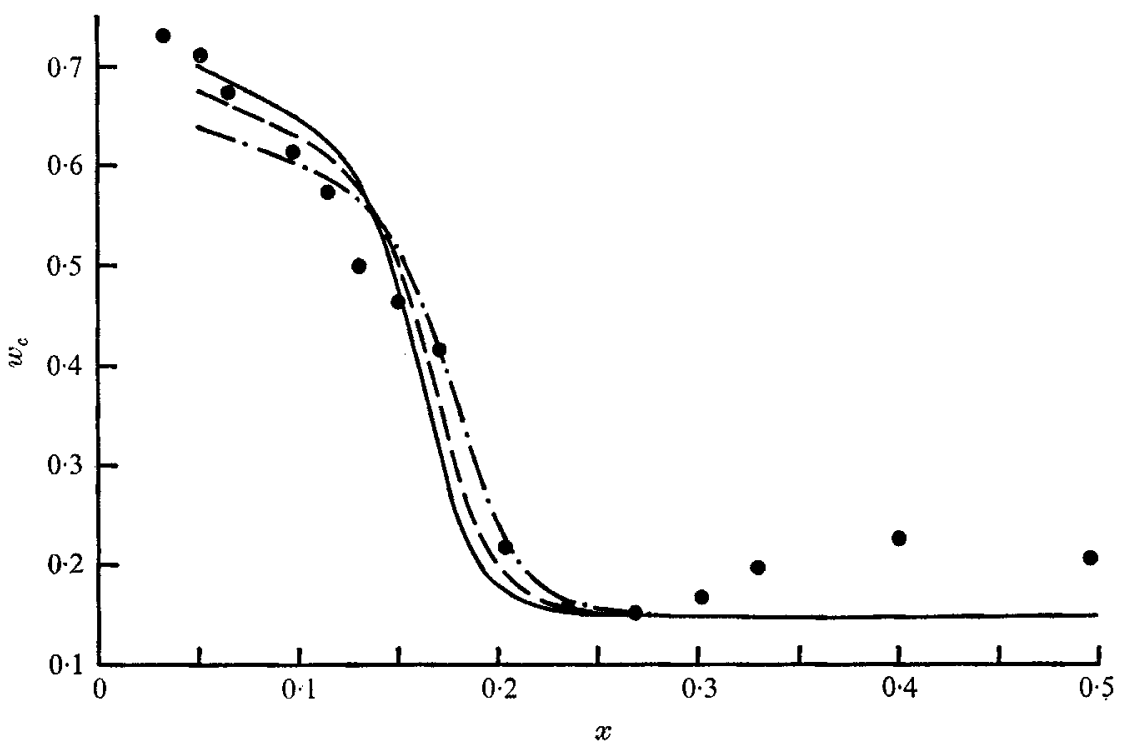

FrguRe 8. Effect of $w_{c 0}$ on the variation of $w_{c}$ at $R=2 \times 10^{5}$ and $E_{u 0}=1 \times 10^{-5}$. $w_{c 0}=0.7 .--, w_{c 0}=0.675 .-., w_{c 0}=0.6386$. experiment of Sato \& Kuriki.

value of $d w_{c} / d x$, and in the delay of the onset of non-linear effect. A few experimental data points are also shown there for comparison. It shows that the experimental data agree better with the result of smaller $w_{c 0}$ in the region downstream of the maximum slope, but a closer agreement is seen with the larger $w_{c 0}$ case for the upstream portion. Figure 9 gives the variations of the integrated fluctuation energy for the three cases. The general variation is again qualitatively unchanged, except for the different locations and levels of the maximum value reached. These differences result from the fact that a higher $w_{c 0}$ implies a thinner wake, and therefore a higher amplification rate locally.

(ii) Effects of $E_{u 0}$. In order to investigate the effect of $E_{u 0}$ on the solutions, a few cases were calculated for $R=2 \times 10^{5}$ with the same value of $w_{c 0}$ but different $E_{u 0}$. Figure 10 shows the variations of $w_{c}$ for three initial values $E_{u 0}=0 \cdot 1,1 \cdot 0$, $3.0 \times 10^{-5}$. The shape of the curves remains practically unchanged. Changing the initial value of $E_{u 0}$ amounts only to a shift of the curve. It is expected from the previous discussions of the physical mechanisms that the magnitude of the disturbance required to cause a sensible deviation of the solution from the undisturbed case is approximately the same over a range of $w_{c}$ where the laminar 


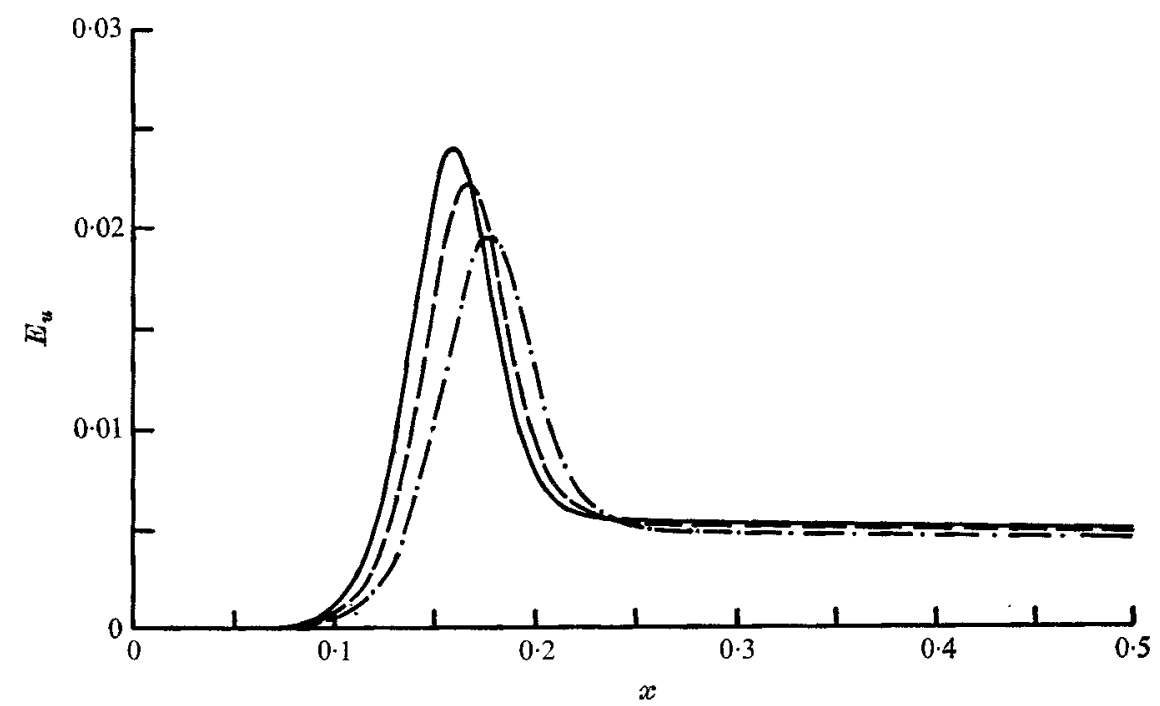

FIGURE 9. Effect of $w_{c 0}$ on the variation of $E_{u}$ at $R=2 \times 10^{5}$ and $E_{u 0}=1 \times 10^{-5}$. $\longrightarrow, w_{c 0}=0 \cdot 7 .---, w_{c 0}=0 \cdot 675 .-.-, w_{c 0}=0.6386$.

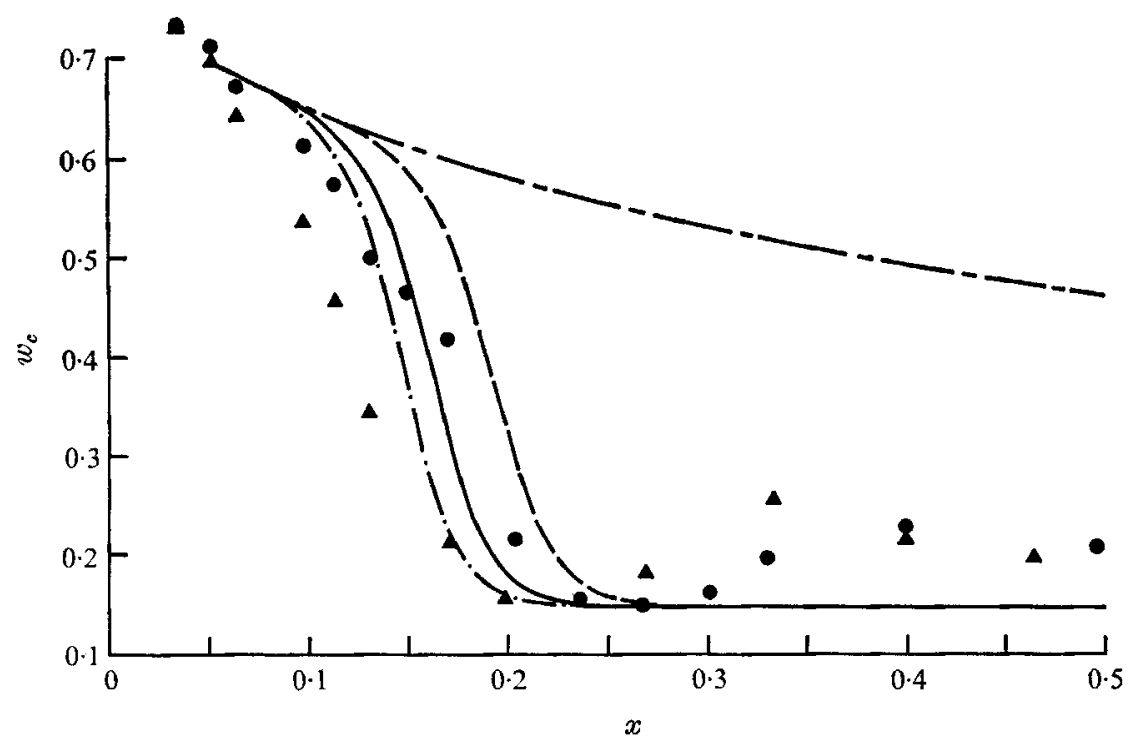

FIgure 10. Effect of $E_{u 0}$ on the variation of $w_{c}$ at $R=2 \times 10^{5}$ and $w_{c 0}=0.7$. - . $E_{u 0}=3 \times 10^{-5}$. -,$E_{u 0}=1 \times 10^{-5}$. - - , $E_{u 0}=1 \times 10^{-6}$. - - , pure laminar wake $\left(E_{u 0} \rightarrow 0\right)$. , measurements of Sato \& Kuriki for a sharp trailing edge model. $\Delta$, measurements of Sato \& Kuriki for a blunt trailing edge model.

viscous term is of the same order. Therefore, a smaller initial magnitude of the disturbance will cause the mean flow to follow the laminar solution for a longer distance before the exponentially amplifying disturbance reaches the significant magnitude. This is further illustrated by the variations of the integrated fluctua- 
tion energy $E_{u}$, shown in figure 11. The shapes are again similar with merely a shift in abscissa. It should be noted that, with the variation of a factor 30 in $E_{u 0}$, the maximum values of $E_{u}$ are nearly the same and the difference in the 'equilibrium' magnitude is negligible. The slight difference in the peak values of $E_{u}$ is caused by the slight change in the magnitude of the laminar viscous term when the interaction becomes important. In principle, if the initial magnitude of the fluctuation is small enough, the solution approaches the steady laminar wake solution corresponding to $E_{u 0} \equiv 0$, which is also shown in figure 10 for comparison.

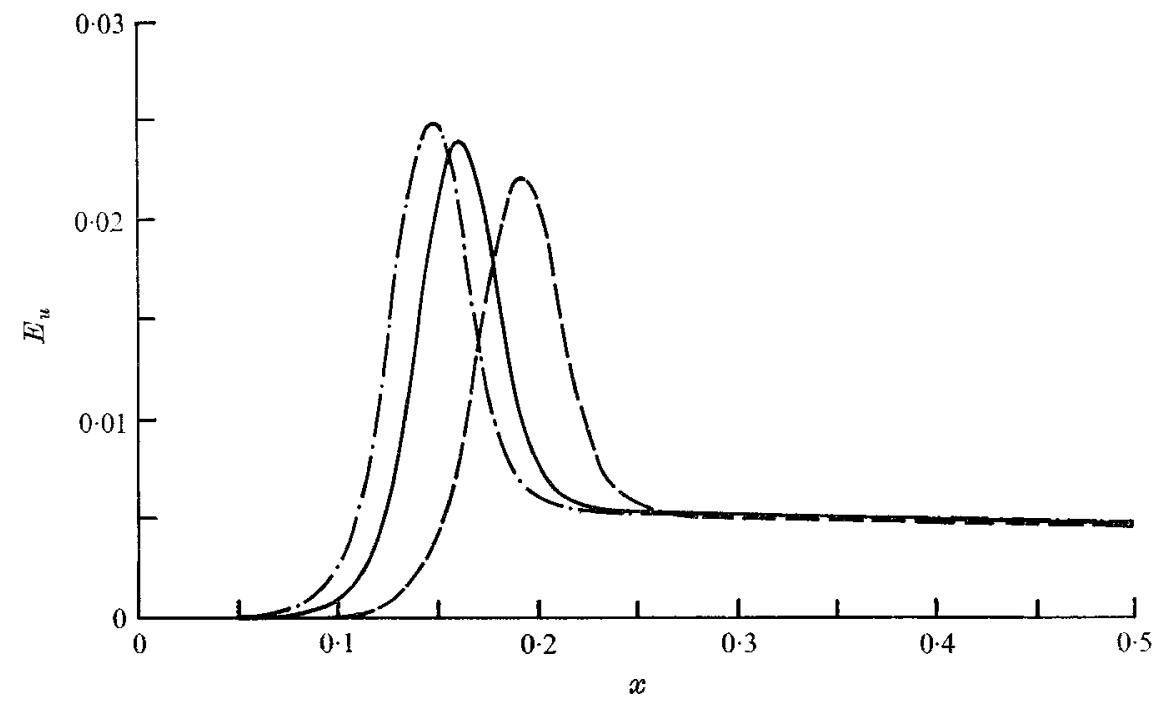

Figure 11. Effect of $E_{u 0}$ on the variation of $E_{u}$ at $R=2 \times 10^{5}$ and $w_{c 0}=0 \cdot 7$. $-\longrightarrow, E_{u 0}=3 \times 10^{-5}$. -,$E_{u 0}=1 \times 10^{-5} .---, E_{u 0}=1 \times 10^{-6}$.

The magnitude of the disturbances existing at the initial stage of the wake depends on many factors which vary from experiment to experiment. These factors include both controllable, e.g. an artificial source of disturbance, and partially controllable, e.g. roughness of the plate, wind tunnel noise level, etc. (If any quantitative calculations are needed, it will be necessary to have some measure of the magnitude of $E_{u 0}$.) This fact is demonstrated by the two sets of experimental data also shown on figure 10. The data points correspond to two different models tested under the same free stream conditions. Model I has a sharp trailing edge, but model II has a blunt one. As expected, a larger $E_{u 0}$ will be associated with model II, which shows the right trend as indicated by the calculations.

\subsection{Effect of Reynolds number}

Since the inviscid Rayleigh equation has been used to obtain the local solutions for the fluctuation, the integrals, $k_{i}$ are universal functions of $w_{c}$ for all Reynolds number. This may seen be from (2.19), where the eigenvalue and the correspond- 
ing eigenfunction are indicated to be functions of $w_{c}$ and $\omega^{*}$. The non-dimensional frequency $\omega^{*}$ is related to the physical angular frequency by

$$
\omega^{*}=\omega b=(b L / U) \times(\text { physical frequency }) .
$$

It was found experimentally by Sato \& Kuriki that the physical frequency of the most unstable sinusoidal fluctuation observed in the linear region of the wake follows a $\frac{3}{2}$ power law as the Reynolds number varies. Furthermore, the halfwidth $b$ is clearly seen from (2.14) to be varying with the drag coefficient, which is proportional to $R^{-\frac{1}{2}}$. Therefore, if we chose to follow the most unstable frequency at various free stream Reynolds numbers, the value of $\omega^{*}$ is a constant which is independent of $R$. Thus, the same functional relations $k_{i}\left(w_{c}\right)$ may be used for different Reynolds numbers that greatly simplify the investigation of the effects of Reynolds number.

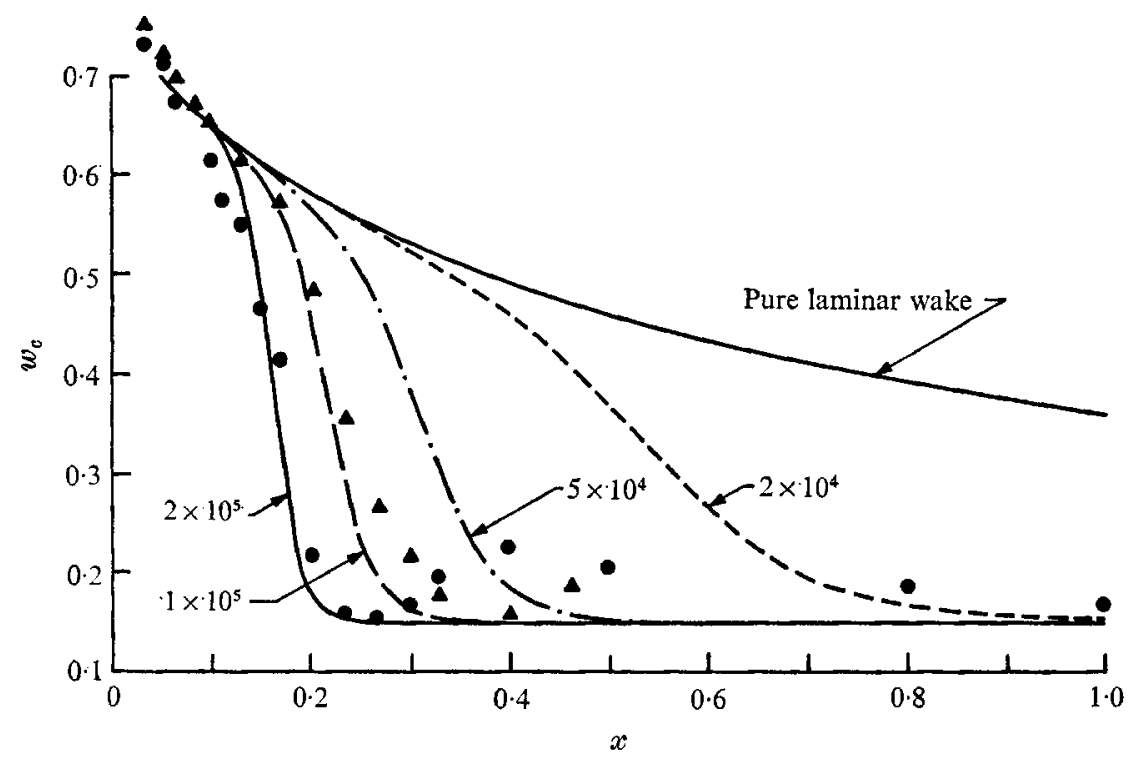

FradRe 12. Reynolds number effect on the variation of $w_{c} \cdot w_{c 0}=0 \cdot 7, E_{u 0}=1 \times 10^{-5}$ for all calculations. The numbers on each curve denote the Reynolds number $R=U L / \nu$. -, measurements of Sato \& Kuriki at $R=2 \times 10^{5}$. $\Lambda$, measurements of Sato \& Kuriki at $R=1 \times 10^{5}$.

Figure 12 shows the variations of $w_{c}$ for four values of Reynolds numbers. The same set of initial values, $w_{c 0}=0.7$ and $E_{u 0}=1 \times 10^{-5}$, has been used in obtaining the solutions, in order to isolate the effects of Reynolds number. The effect on the solution is somewhat similar to the effect of changing the initial magnitude of the fluctuation $E_{u 0}$. In fact, it may be seen from $(2.27)$ that the ratio of the Reynolds stresses term to the laminar viscous term is of the order $|A|^{2} \sqrt{ } R$. Hence, the effect of $R$ on the beginning of transition is similar to the effect of $E_{u 0}$. However, the effect of $R$ differs through its persist ientnfluence over the whole non-linear region other than a mere shift of the abscissa. When the Reynolds 
number decreases, the viscous damping term becomes more important in the non-linear region, therefore the overall transition becomes smoother.

Also shown in figure 12 are two sets of experimental data of Sato \& Kuriki at $R=2 \times 10^{5}, 1 \times 10^{5}$ correspondingly. The agreement in the trend, and even the quantitative effect, are fairly good considering that, if $E_{u 0}=1 \times 10^{-5}$ were correct for the case of $R=2 \times 10^{5}$, the same value of $E_{u 0}$ might have been too large for the smaller Reynolds number case, due to a relatively lower tunnel noise level.

Because of the persistent influence of the Reynolds number on the interaction, its effect on the fluctuation energy is more pronounced than the effect of $E_{u 0}$. The result is shown in figure 13, where it may also be noted that the final equilibrium amplitudes reached are different, because of the effect of Reynolds number appearing through the viscous dissipating term in (2.16). This result is also different from the effect of $E_{u 0}$, which leaves the final equilibrium amplitude of the fluctuation practically unchanged.

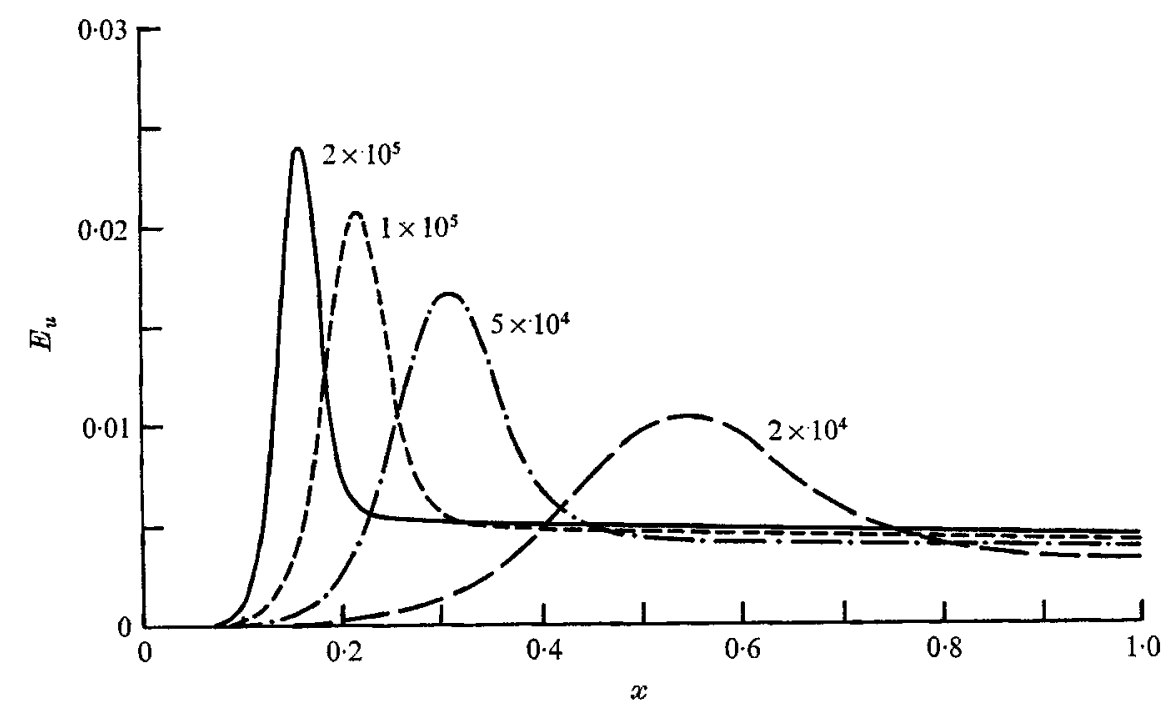

FIGURE 13. Reynolds number effect on the variation of $E_{u} \cdot w_{c 0}=0 \cdot 7, E_{u 0}=1 \times 10^{-5}$ for all calculations. The numbers on each curve denote the Reynolds number $R$.

\subsection{Effect of the viscous dissipation of the fluctuation}

The effect of the viscous dissipation of the fluctuation on the development of the disturbances was studied simply by setting $I_{8} \equiv 0$. Comparison is made in figures 14 and 15 for the case of $R=2 \times 10^{5}$. With identical initial conditions, figure 14 shows that the effect of the viscous term in the fluctuation energy equation on the mean flow is quite small as one would expect. Figure 15 shows that, if the viscous dissipation term is absent, a final equilibrium amplitude (namely, another laminar oscillatory flow) is reached. However, because of the effect of the viscous dissipation, this equilibrium condition cannot maintain itself, and the amplitude decays slowly as it proceeds further downstream, to account for the energy loss. 


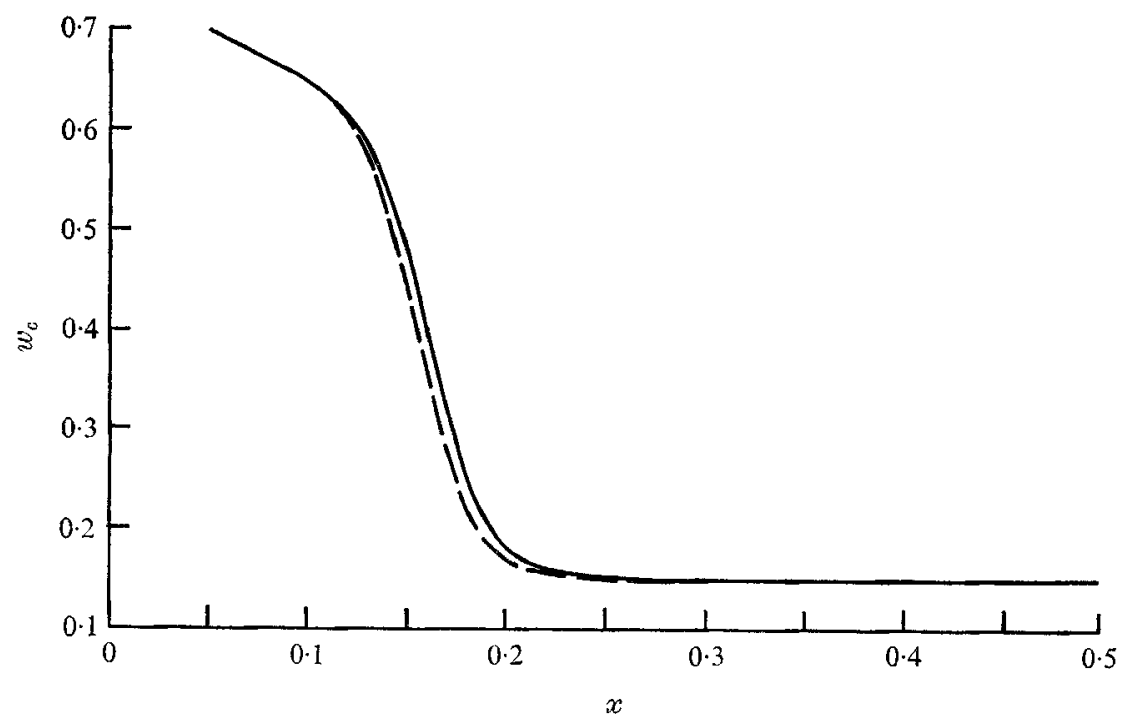

Frgure 14. Effect of the viscous dissipation term $T_{v f}$ due to the fluctuation on the variation of $w_{c} . R=2 \times 10^{5}, E_{u 0}=1 \times 10^{-5}, w_{c 0}=0.7$. - - non-zero viscous dissipation. -- , zero viscous dissipation.

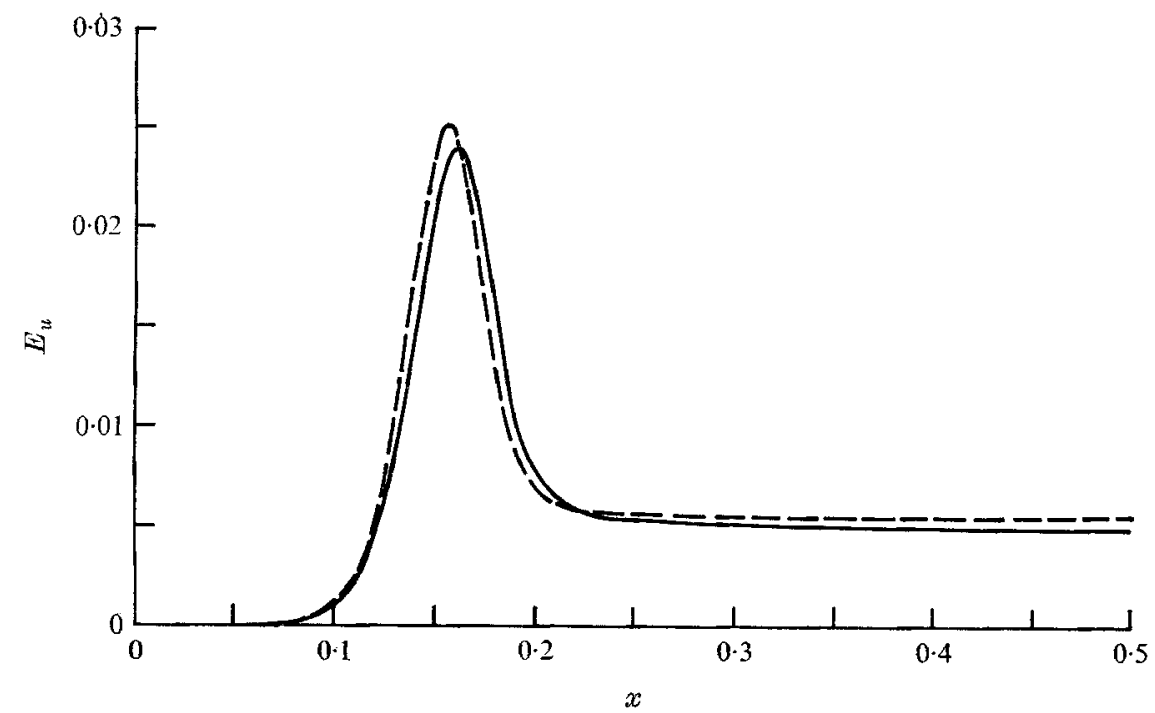

Figure 15. Effect of the viscous dissipation term $T_{v f}$ due to the fluctuation on the variation of $E_{u} . R=2 \times 10^{5}, E_{u 0}=1 \times 10^{-5}, w_{c 0}=0 \cdot 7$. $\longrightarrow$, non-zero viscous dissipation. - - , zero viscous dissipation. 


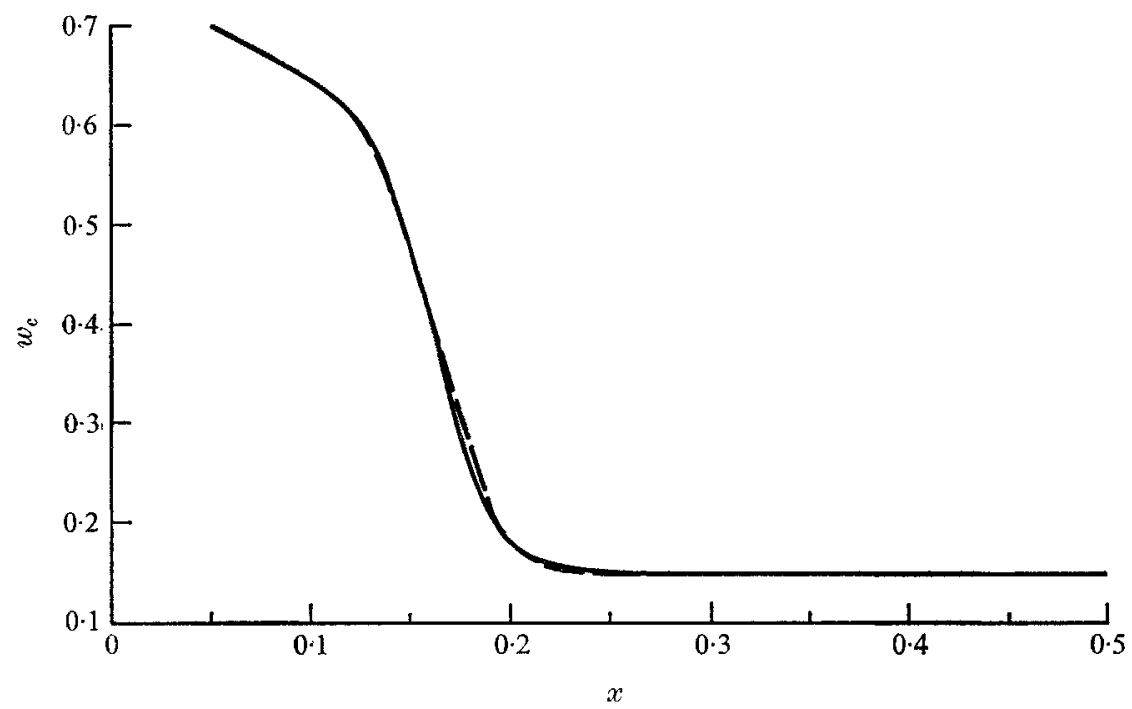

FiguRe 16. Effect of decoupling $b$ from $w_{c}$ on the variation of $w_{c} . R=2 \times 10^{5}$, $E_{u 0}=1 \times 10^{-5}, w_{\mathrm{c} 0}=0 \cdot 7 .-, b=b\left(w_{c}\right) .--, b=b\left(w_{\mathrm{c}},|A|^{2}\right)$.

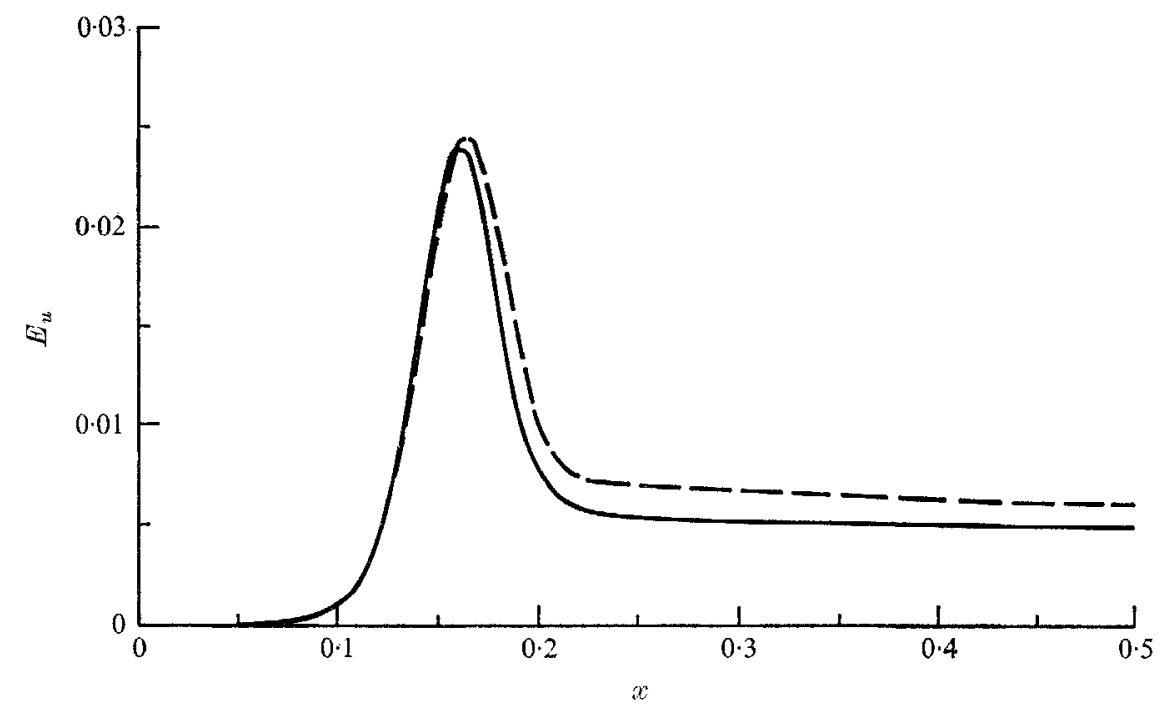

Figure 17. Effect of decoupling $b$ from $w_{c}$ on the variation of $E_{u} \cdot R=2 \times 10^{5}$, $E_{u 0}=1 \times 10^{-5}, w_{00}=0.7 . \longrightarrow, b=b\left(w_{c}\right),--, b=b\left(w_{c},|A|^{2}\right)$.

\subsection{Effect of coupling $b$ to $w_{c}$}

The assumption of neglecting the contribution from the fluctuating components in the integrated momentum equation, which leads to a simple relation (2.20) between $b$ and $w_{c}$, is now examined. From the calculated results, it is found that the terms, which have been neglected to arrive at the expression (2.20), are indeed small compared to the remaining terms in the initial stage, but increase to about 
$20 \%$ of the sum of the remaining terms when the amplitude of the fluctuation reaches a maximum. It is therefore desirable to investigate the effect of using the full integral momentum equation.

An exact formulation will require the computation of the integrals $k_{i}$ as functions of two parameters, $b$ and $w_{c}$, since $\omega^{*}=\omega b$. To simplify the analysis, we assume that the integrals may still be taken as functions of $w_{c}$ only, but the integral momentum (2.14) is used instead of the approximated (2.20). Then, the wake half-width $b$ is a function of $w_{c}$, as well as the amplitude of the fluctuation.

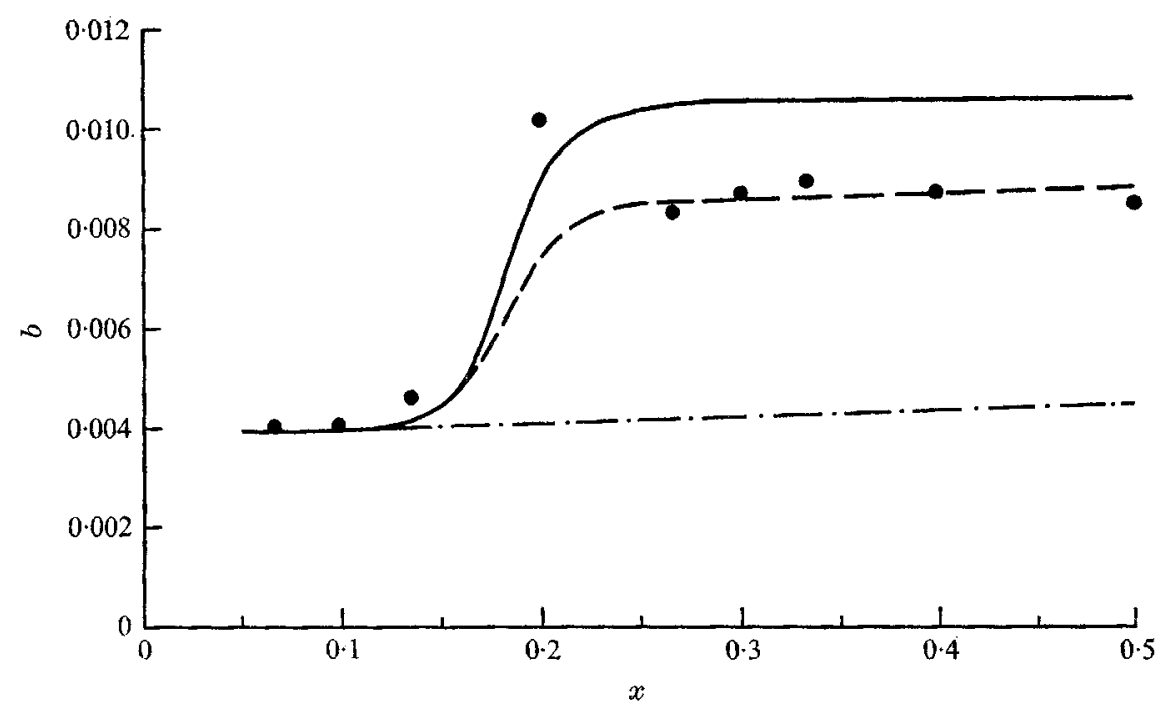

Figure 18. Comparison of the non-dimensional wake half-width $b$ at $R=2 \times 10^{5}$. $E_{u 0}=1 \times 10^{-5}, w_{c 0}=0.7$. -,$b=b\left(w_{c}\right) .--, b=b\left(w_{c},|A|^{2}\right)$. ○, measurements of Sato \& Kuriki. -..- - pure laminar wake.

The results of such an integration are shown in figures 16-18 for the case of $R=2 \times 10^{5}$. It is clear that the effect on the mean flow velocity variation is quite small. Since a relatively thinner wake is obtained when $b$ is decoupled from $w_{c}$, it affects the variation of the fluctuation energy and the final level approached. The variation of wake half-width $b$ for the two cases is shown in figure 18, together with the measured results of Sato \& Kuriki. The decoupled result agrees better with the measurements, especially in the 'equilibrium' wake width.

\section{Concluding remarks}

Based on the comparison of the numerical calculations with the experimental data, the present approach brings out the essential features of the non-linear interaction mechanisms in a laminar wake. The theory shows that the relatively rapid deviation of the mean flow velocity along the wake axis from the prediction for a steady laminar wake, and the rapid growth of the wake width observed experimentally in the transition region, are the consequences of the non-linear 
effects induced by the finite amplitude disturbances. The streamwise variation of the magnitude of the fluctuation is in favourable agreement with the relative development observed experimentally. The decrease in magnitude of the fluctuation in the latter stage of the non-linear region, which is abnormal in view of the linear stability theory, is found to be a consequence of the wake growth.

Although the present study of the interaction of a single frequency fluctuation with the mean flow through the Reynolds stresses provides a result that is consistent with most of the observed phenomena in the experiment of Sato \& Kuriki, another important aspect of the non-linear mechanism, which resulted from the quadratic terms $\chi_{1}$ and $\chi_{2}$ in the fluctuation equations (2.4), may not be completely ignored. These terms are responsible for the generation of the higher harmonics and the modification of the lower ones. The appearance of the second harmonic, and the rather drastic variation of the distributions of the magnitude and phase of the fundamental mode in the non-linear region of the experiments of Sato \& Kuriki, calls for the inclusion of such effects. (This will be reported in a forthcoming paper.)

In the spirit of an integral approach, the detail distribution of the fluctuation should not be of much concern to the assessment of the results. However, any drastic deviation from the assumed profile shape should at least lead to more caution in the interpretation of outcomes. The agreement of the measured distribution in the linear region with the solution of the inviscid Rayleigh equation has been well demonstrated by the temporal mode calculation of Sato \& Kuriki. The same degree of agreement is shown by the present spatial mode calculation. However, the measured distributions for $x>0 \cdot 2$ deviate substantially from the linear profiles. Inclusion of the second harmonic, and modification of the fundamental mode using the quasi-parallel mean flow, did not resolve this difficulty. It is our conjecture that the deviations in the distribution result, in a large part, from the quasi-parallel flow assumption, which becomes increasingly inadequate in the downstream direction.

Finally, the integral method of solution suggested in this paper provides a means for extending and applying the classical hydrodynamic stability to a real problem. A similar procedure can be applied to flows of inviscid nature and governed by two-dimensional disturbances.

This work was carried out under the sponsorship and with the financial support of the U.S. Army Research Office and the Advanced Research Projects Agency under Contract DA-31-124-ARO(D)-33, part of Project DEFENDER sponsored by the Advanced Research Projects Agency.

\section{REFERENCES}

GOLD, H. 1963 Stability of laminar wakes. Ph.D. Thesis, California Institute of Technology.

Goldsters, S. 1933 On the two-dimensional steady flow of a viscous fluid behind a solid body. Part. I. Proc. Roy. Soc. Lond. A 142, 545.

Kuwabara, S. 1967 Nonlinear instability of plane Couette flow. Phys. of Fluid. Supplement Copy on Boundary Layer and Turbulence 10:9, Part IT, S 115. 
LANDAU, L. 1944 Stability of tangential discontinuity in compressible fluid. C.R. (Doklady) Acad. Sci. U.S.S.R. 44, 311.

McKoes, C. H. 1955 On the stability of a laminar wake. ARC CP 303.

Meksyn, D. \& Stuart, J. T. 1951 Stability of viscous motion between parallel planes for finite disturbances. Proc. Roy. Soc. Lond. A 208, 517.

Sato, H. \& KuRIKI, K. 1961 The mechanism of transition in the wake of a thin flat plate placed parallel to a uniform flow. J. Fluid Mech. 11, 321.

Stuart, J. T. 1956 On the role of the Reynolds stresses in stability theory. J. Aero. Sci. 23, 86.

Struart, J.T. 1958 On the non-linear mechanics of hydrodynamic stability. $J$. Fluid Mech. 4, 1.

STUART, J.T. 1960 On the non-linear mechanics of wave disturbances in stable and unstable parallel flows. Part 1 . The basic behaviour in plane Poiseuille flow. $J$. Fluid Mech. 9, 353.

Taylor, G. I. 1923 Stability of a viscous liquid contained between two rotating cylinders. Phil. Trans. Roy. Soc. Lond. A 223, 289.

WATSON, J. 1960 On the non-linear mechanies of wave disturbances in stable and unstable parallel flows. Part 2. The development of a solution for plane Poiseuille flow and for plane Couette flow. J. Fluid Mech. 9, 371.

Watson, J. 1962 On spatially-growing finite disturbances in plane Poiseuille flow. J. Fluid Mech. 14, 211. 